Research Article

\title{
Numerical Approximation of Generalized Burger's-Fisher and Generalized Burger's-Huxley Equation by Compact Finite Difference Method
}

\author{
Ravneet Kaur $\mathbb{D}^{1}$, Shallu $\mathbb{D}^{1},{ }^{1}$ Sachin Kumar $\mathbb{D}^{2},{ }^{2}$ and V. K. Kukreja $\mathbb{D}^{1}$ \\ ${ }^{1}$ Department of Mathematics, SLIET, Longowal, 148106 Punjab, India \\ ${ }^{2}$ Department of Mathematics and Statistics, Central University of Punjab, Bathinda, 151001 Punjab, India \\ Correspondence should be addressed to V. K. Kukreja; vkkukreja@gmail.com
}

Received 16 May 2021; Accepted 7 October 2021; Published 9 November 2021

Academic Editor: Giuseppe Pellicane

Copyright (C) 2021 Ravneet Kaur et al. This is an open access article distributed under the Creative Commons Attribution License, which permits unrestricted use, distribution, and reproduction in any medium, provided the original work is properly cited.

\begin{abstract}
In this work, computational analysis of generalized Burger's-Fisher and generalized Burger's-Huxley equation is carried out using the sixth-order compact finite difference method. This technique deals with the nonstandard discretization of the spatial derivatives and optimized time integration using the strong stability-preserving Runge-Kutta method. This scheme inculcates four stages and third-order accuracy in the time domain. The stability analysis is discussed using eigenvalues of the coefficient matrix. Several examples are discussed for their approximate solution, and comparisons are made to show the efficiency and accuracy of CFDM6 with the results available in the literature. It is found that the present method is easy to implement with less computational effort and is highly accurate also.
\end{abstract}

\section{Introduction}

The excerpt approximation of the Navier-Stokes equation is represented by a prominent nonlinear mathematical model known as Burger's equation. It is the perfect combination of advection and diffusion terms. This equation was introduced by Bateman [1]. Later, Burger [2] extensively worked on this problem, considering the turbulence effect and the statistical aspects. Burger's equation describes the process of simulating shock wave phenomena, dispersion in a porous medium, heat conduction, diffusion flow, modeling of gas dynamics, traffic flow, propagation and reflection of the nonlinear fluid, boundary layer flow, electrohydrodynamics, sound waves, oil reservoir simulation, etc. The spreading of any species due to the favorable environment of the invasive species or predicting the pattern of spreading was an important issue in the early twenties. The great researcher Fisher [3] proposed a model for the temporal and spatial propagation, depicting the wave of increase in gene frequency in an infinite medium and termed it as Fisher's equation. It represents the biological processes, ecological systems, pattern formation, etc. Petrovskii and Shigesada [4] combined both the models by assuming that the distribution of species is symmetrical and the environment is homogeneous. The following $1 \mathrm{D}$ equation was proposed:

$$
\frac{\partial z}{\partial t}=\frac{\partial^{2} z}{\partial x^{2}}+\mathfrak{F}\left(x, t, z, z_{x}\right), \quad \text { in } \Phi=\Phi_{x} \times \Phi_{t},
$$

with the initial and boundary conditions:

$$
\begin{array}{cc}
z=z^{0}, & \text { in } \bar{\Phi}_{x} \times t_{0}, \\
\mathfrak{B} z=\Omega, & \text { on } \partial \Phi_{x} \times \bar{\Phi}_{t},
\end{array}
$$

where $\Phi_{x}=(a, b), \Phi_{t}=(0, t)$, and $\mathfrak{B}$ is the boundary operator. A mathematical model for $\mathfrak{f}\left(x, t, z, z_{x}\right)=-\beta z^{\delta} z_{x}+\gamma z\left(1-z^{\delta}\right)$ in (1) with the above conditions is known as the generalized Burger's-Fisher (gBF) equation and is expressed as follows:

$$
\frac{\partial z}{\partial t}-\frac{\partial^{2} z}{\partial x^{2}}+\beta z^{\delta} \frac{\partial z}{\partial x}-\gamma z\left(1-z^{\delta}\right)=0, \quad 0 \leq x \leq 1, t \geq 0,
$$

subject to the initial condition: 


$$
z(x, 0)=\left[\frac{1}{2}+\frac{1}{2} \tanh \left(\frac{-\beta \delta x}{2(1+\delta)}\right)\right]^{1 / \delta}
$$

and the boundary conditions:

$z(0, t)=\left(\frac{1}{2}+\frac{1}{2} \tanh \left(\frac{\beta \delta}{2(1+\delta)}\left[\left(\frac{\beta^{2}+\gamma(1+\delta)^{2}}{\beta(1+\delta)} t\right)\right]\right)\right)^{1 / \delta}, \quad t \geq 0$

$z(1, t)=\left(\frac{1}{2}+\frac{1}{2} \tanh \left(\frac{-\beta \delta}{2(1+\delta)}\left[1-\left(\frac{\beta^{2}+\gamma(1+\delta)^{2}}{\beta(1+\delta)} t\right)\right]\right)\right)^{1 / \delta}$

where $\beta, \gamma$, and $\delta$ are the constants. The choice of the value of these constants reduces the model to different forms of PDEs. For $\gamma=0$, it reduces to the generalized Burger's equation. Taking $\beta=0$, it becomes the generalized Fisher's equation. The exact solution of Equation (3) was given by Chen and Zhang [5] as follows:

$$
z(x, t)=\left(\frac{1}{2}+\frac{1}{2} \tanh \left(\frac{-\beta \delta}{2(1+\delta)}\left[x-\left(\frac{\beta^{2}+\gamma(1+\delta)^{2}}{\beta(1+\delta)} t\right)\right]\right)\right)^{1 / \delta}
$$

Over the past many years, work has been done for the explicit solution of Equation (3). Numerical methods provide a tool for the physical behaviour of the system, although theoretical results are available in the literature. Sari et al. [6] applied the compact finite difference method along with the third-order total variation-diminishing Runge-Kutta scheme in the time domain. Zhao et al. [7] implemented the pseudospectral method using the time discretization by Crank-Nicolson as well as the leapfrog scheme and space discretization by LegendreGalerkin and Chebyshev-Gauss-Lobatto for nodes. Mohammadi [8] proposed the exponential spline and finite difference approximations. Tatari et al. [9] analyzed the radial basis function collocation technique with the predictor-corrector method. Malik et al. [10] discussed the hybridization of the Exp-function method with the nature-inspired algorithm. Yadav and Jiwari [11] analyzed the finite element analysis with the existence and uniqueness of the weak solution using Galerkin's finite element method. Macias-Diaz and Gonzalez [12] implemented the finite difference method. Soori [13] obtained the exact solution of the Burger's-Fisher equation using the variational iteration method and homotopy perturbation method. An exponential time differencing scheme using the method of lines was developed by Bratsos and Khaliq [14]. Gurbuz and Sezer [15] discussed the modified Laguerre matrix-collocation method.

The significance and various applications motivated the researchers to compute the analytical and numerical solutions of the Burger's-Fisher equation. Recently, the dynamical behaviour and exact parametric representations of the traveling wave solutions under different parametric conditions have been discussed by $\mathrm{Li}$ [16]. In the findings, the exact monotonic and nonmonotonic kink wave solutions, two-peak solitary wave solutions, and periodic wave solu- tions, as well as unbounded traveling wave solutions have been obtained. Onyejekwe et al. [17] applied a boundary integral element-based numerical technique, in which the boundary and domain values calculate the fundamental integral inside the domain. The domain integrals due to nonlinearity are considered for computing the solution. Investigation of the global existence and uniqueness of a periodic wave solution has been conducted by Zhang et al. [18].

Another important nonlinear equation, describing the interaction between reaction mechanism, convection effect, and diffusion transport is the 1D generalized Burger'sHuxley (gBH) equation, for which $\mathfrak{f}\left(x, t, z, z_{x}\right)=-\beta z^{\delta} z_{x}+\gamma$ $z\left(1-z^{\delta}\right)\left(z^{\delta}-\eta\right)$. The equation is expressed as follows:

$\frac{\partial z}{\partial t}-\frac{\partial^{2} z}{\partial x^{2}}+\beta z^{\delta} \frac{\partial z}{\partial x}=\gamma z\left(1-z^{\delta}\right)\left(z^{\delta}-\eta\right), \quad a \leq x \leq b, t \geq 0$

The parameters $\beta, \gamma$, and $\delta$ are the constants and parameter $\eta \in(0,1)$. The initial and boundary conditions are as follows:

$$
\begin{aligned}
z(x, 0) & =\left(\frac{\eta}{2}+\frac{\eta}{2} \tanh \left(A_{1} x\right)\right)^{1 / \delta}, \\
z(a, t) & =\left[\frac{\eta}{2}+\frac{\eta}{2} \tanh \left(A_{1}\left(a-A_{2} t\right)\right)\right]^{1 / \delta}, z(b, t) \\
& =\left[\frac{\eta}{2}+\frac{\eta}{2} \tanh \left(A_{1}\left(b-A_{2} t\right)\right)\right]^{1 / \delta} .
\end{aligned}
$$

The exact solution derived by Wang [19], using nonlinear transformations, is reproduced hereunder:

$$
z(x, t)=\left[\frac{\eta}{2}+\frac{\eta}{2} \tanh \left(A_{1}\left(x-A_{2} t\right)\right)\right]^{1 / \delta},
$$

where

$$
\begin{aligned}
A_{1} & =\eta \delta\left(\frac{-\beta+\sqrt{\beta^{2}+4 \gamma(1+\delta)}}{4(\delta+1)}\right), A_{2} \\
& =\frac{\beta \eta}{\delta+1}-\frac{(1-\eta+\delta)\left(-\beta+\sqrt{\beta^{2}+4 \gamma(\delta+1)}\right)}{2(\delta+1)} .
\end{aligned}
$$

For $\gamma=0$, the above model conforms to the generalized Burger's equation, and considering $\beta=0$ and $\delta=1$, the Huxley equation [20] is obtained. For $\beta=0, \gamma=1$, and $\delta=1$, it corresponds to the Fitzhugh-Nagoma equation [21]. Yefimova and Kudryashov [22] applied the Hopf-Cole transformation for solving the $\mathrm{gBH}$ equation. The Adomian decomposition method was implemented by Ismail et al. [23]. Gao and Zhao [24] proposed the Exp-function method for a series of exact solutions of the $\mathrm{gBH}$ equation. A high-order difference scheme using Taylor's series expansion was presented by Sari et al. [25]. Celik [26] introduced a numerical method based on the Haar wavelet approach. 
Zhang et al. [27] reduced the Burger's-Huxley and Burger's-Fisher equations into first-order systems and then applied the discontinuous Galerkin method. A numerical scheme based on the finite differences for time integration and cubic B-spline for space integration was proposed by Mohammadi [28]. A fourth-order finite difference method was implemented by Bratsos [29] in a two-time level recurrence relation for the solution of the gBH equation. El-Kady et al. [30] discussed the methods based on cardinal Chebyshev and Legendre basis functions with the Galerkin method, Gauss quadrature formula, and El-Gendi method to convert the problem into ordinary differential equations. Technique based on modified cubic B-spline as the basis function with differential quadrature method was discussed by Singh et al. [31]. The nonstandard finite difference method was analyzed by Zibaei et al. [32]. Bukhari [33] applied local radial basis function differential collocation method. Macias-Diaz [34] used the explicit exponential method. Gilani and Saeed [35] applied the CAS wavelet in conjunction with the Picard technique. Cardinal B-spline wavelet numerical method was used by Shiralashetti and Kumbinarasaiah [36]. A technique based on the hyperbolic-trigonometric tension B-spline method was applied by Alinia and Zarebnia [37]. Loyinmi and Akinfe [38] proposed an algorithm using the coupling of the Elzaki transform with the homotopy perturbation method.

Recently, the exact solution has been computed by Kushner and Matviichuk [39] using the theory of finitedimensional dynamics. Shukla and Kumar [40] applied the numerical scheme based on the Crank-Nicolson finite difference method in collaboration with the Haar wavelet analysis, to obtain the numerical solution. A feed-forward artificial neural network technique is applied by Panghal and Kumar [41] in which the constructed error function is minimized using the quasi-Newton algorithm.

Based on the traditional finite difference approximations, Lele [42] proposed well-regulated compact schemes to provide a better representation of shorter proportionate lengths. Many researchers have extended the compact finite difference scheme for linear/nonlinear differential equations, partial differential equations having Dirichlet or Neumann boundary conditions. Ansari et al. [43] implemented the CFD6 scheme for free vibration phenomena of nanobeams in an elastic medium. A similar scheme for incompressible Navier-Stokes and scalar transport equation was analyzed by Boersma [44], a reaction-diffusion equation with delay was approximated by Li et al. [45] and the modified Burger's equation by Kaur et al. [46].

In this work, a numerical scheme based on the sixthorder compact finite difference method (CFDM6) followed by the strong stability-preserving Runge-Kutta method (SSP-RK43) for time integration is used to solve gBF and gBH equations. The advantage of CFDM6 with the SSPRK43 method is that it computes the results at more mesh points, giving a better approximate solution. The proposed method gives the sixth order of convergence in the spatial domain and the third order in the temporal domain. The proposed method is easy to implement and has less computational cost. The future scope of the method is to solve various arduous linear and nonlinear PDEs.
The paper is organized as follows: in Section 2, first- and second-order spatial derivatives of the CFDM6 are derived. In Section 3, the proposed method is implemented followed by SSP-RK43. In Section 4, convergence is discussed. In Section 5, stability analysis for the proposed scheme is presented. In Section 6, several test problems are discussed to demonstrate and justify the applicability of the proposed scheme. In Section 7, the conclusion explaining the efficiency of CFDM6 is given.

\section{Compact Finite Difference Method}

The spatial domain $\phi_{x}=(a, b)$ is divided into uniform mesh with step iteration $x_{i}=a+i h, i=0,1,2, \cdots, N, h=(b-a) / N$ and for time domain $\phi_{t}=\left(t_{0}, t\right)$, with $t_{0}=0$, a uniform step of size $\Delta t=t^{j+1}-t^{j}$ such that $t^{j}=t_{0}+j \Delta t, j=0,1,2, \cdots$, is followed. The method for calculating first-order and second-order derivatives using the compact finite difference scheme is given hereunder.

2.1. Spatial Derivatives of First Order. The first-order spatial derivatives for CFDM6 at the inner nodes are calculated as follows [42]:

$$
\varphi z_{i-1}^{\prime}+z_{i}^{\prime}+\varphi z_{i+1}^{\prime}=\chi\left(\frac{z_{i+2}-z_{i-2}}{4 h}\right)+\psi\left(\frac{z_{i+1}-z_{i-1}}{2 h}\right) .
$$

For the optimality of the scheme with higher-order accuracy, consider $\varphi=1 / 3$ representing the implicit form of the first-order derivative. The unknown parameters on the other side are calculated by the relation $\chi=(1 / 3)(4 \varphi-1)$ and $\psi$ $=(2 / 3)(2+\varphi)$. By simple calculation, Equation (13) reduces to a sixth-order tridiagonal matrix as a linear system of equations given below with truncation error $(4 / 7 !) h^{6} z_{i}^{(7)}$ :

$$
z_{i-1}^{\prime}+3 z_{i}^{\prime}+z_{i+1}^{\prime}=\frac{-z_{i-2}-28 z_{i-1}+28 z_{i+1}+z_{i+2}}{12 h}, \quad i=2,3, \cdots, N-2 .
$$

For the value of the derivative at $x_{0}, x_{1}, x_{N-1}$, and $x_{N}$, one-sided forward and backward schemes have been implemented, which produce following results:

$$
\begin{aligned}
& z_{0}^{\prime}+5 z_{1}^{\prime}=\frac{1}{60 h}\left(-197 z_{0}-25 z_{1}+300 z_{2}-100 z_{3}+25 z_{4}-3 z_{5}\right), \\
& 2 z_{0}^{\prime}+11 z_{1}^{\prime}+2 z_{2}^{\prime}=\frac{1}{12 h}\left(-80 z_{0}-35 z_{1}+136 z_{2}-28 z_{3}+8 z_{4}-z_{5}\right), \\
& 2 z_{N-2}^{\prime}+11 z_{N-1}^{\prime}+2 z_{N}^{\prime}=\frac{1}{12 h}\left(z_{N-5}-8 z_{N-4}+28 z_{N-3}\right. \\
& \left.\quad-136 z_{N-2}+35 z_{N-1}+80 z_{N}\right), \\
& 5 z_{N-1}^{\prime}+z_{N}^{\prime}=\frac{1}{60 h}\left(3 z_{N-5}-25 z_{N-4}+100 z_{N-3}\right. \\
& \left.\quad-300 z_{N-2}+25 z_{N-1}+197 z_{N}\right) .
\end{aligned}
$$

The relations (14) and (15) can be represented in the form of a matrix system as

$$
\mathbb{A} z^{\prime}=\mathbb{B} z
$$


where

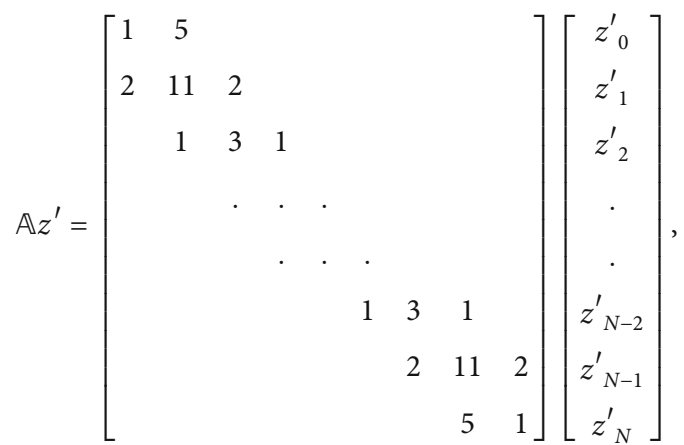

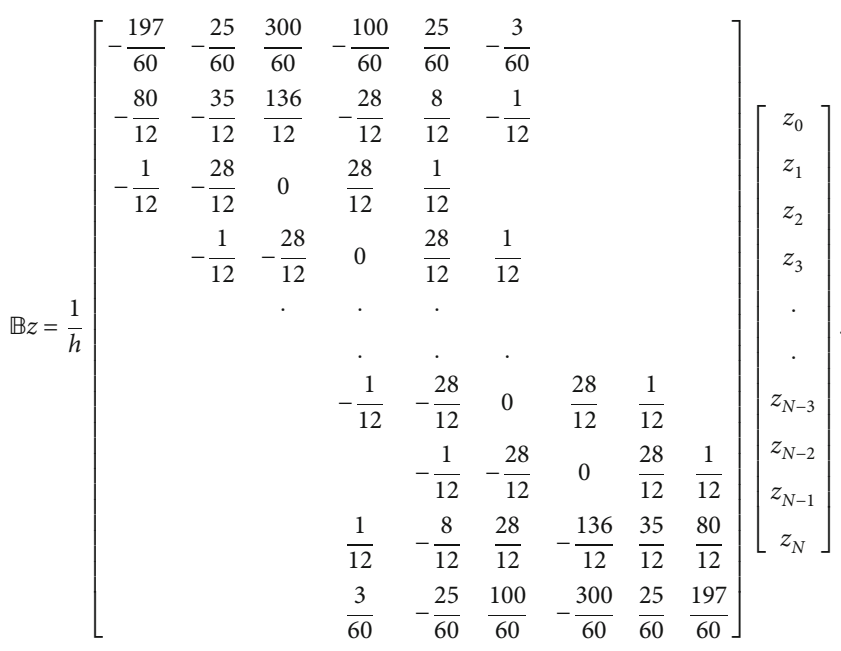

2.2. Spatial Derivatives of Second Order. Similarly, the second-order derivative is calculated as

$\tau z^{\prime \prime}{ }_{i-1}+z^{\prime \prime}{ }_{i}+\tau z^{\prime \prime}{ }_{i+1}=\sigma\left(\frac{z_{i+2}-2 z_{i}+z_{i-2}}{4 h^{2}}\right)+\varsigma\left(\frac{z_{i+1}-2 z_{i}+z_{i-1}}{h^{2}}\right)$.

For $\tau=0$, this equation represents the explicit method to calculate the derivative, and for $\tau=1 / 10$, it will represent the implicit scheme of the second-order derivative. The unknown constants on the R.H.S. are calculated as $\varsigma=(4 / 3)$ $(1-\tau)$ and $\sigma=(1 / 3)(-1+10 \tau)$. This reduces Equation (18) to a tridiagonal system as follows:

$$
z^{\prime \prime}{ }_{i-1}+10 z^{\prime \prime}{ }_{i}+z^{\prime \prime}{ }_{i+1}=\frac{12}{h^{2}}\left(z_{i+1}-2 z_{i}+z_{i-1}\right) .
$$

For the boundary points, one-sided forward and backward schemes have been implemented, which gives the following results:

$10 z^{\prime \prime}{ }_{0}+z^{\prime \prime}{ }_{1}=\frac{12}{h^{2}}\left(\frac{115}{36} z_{0}-\frac{1555}{144} z_{1}+\frac{89}{6} z_{2}-\frac{773}{72} z_{3}+\frac{151}{36} z_{4}-\frac{11}{16} z_{5}\right)$,

$$
\begin{aligned}
{z^{\prime \prime}}^{\prime}{ }_{N-1}+10 z^{\prime \prime}{ }_{N}= & \frac{12}{h^{2}}\left(\frac{115}{36} z_{N}-\frac{1555}{144} z_{N-1}+\frac{89}{6} z_{N-2}\right. \\
& \left.-\frac{773}{72} z_{N-3}+\frac{151}{36} z_{N-4}-\frac{11}{16} z_{N-5}\right) .
\end{aligned}
$$

The second-order derivative can be written in the matrix form as

$$
\begin{aligned}
& \mathbb{C} z^{\prime \prime}=\mathbb{D} z, \\
& \mathbb{C} z^{\prime \prime}=\left[\begin{array}{ccccccccc}
10 & 1 & & & & & & \\
1 & 10 & 1 & & & & & & \\
& 1 & 10 & 1 & & & & \\
& & \cdot & \cdot & \cdot & & & & \\
& & & \cdot & \cdot & \cdot & & & \\
& & & & \cdot & \cdot & \cdot & & \\
& & & & & 1 & 10 & 1 & \\
& & & & & 1 & 10 & 1 \\
& & & & & & 1 & 10
\end{array}\right],\left[\begin{array}{c}
z^{\prime \prime}{ }_{0} \\
z^{\prime \prime}{ }_{1} \\
z^{\prime \prime}{ }_{2} \\
\cdot \\
\cdot \\
\cdot \\
z^{\prime \prime}{ }_{N-2} \\
z^{\prime \prime}{ }_{N-1} \\
z^{\prime \prime}{ }_{N}
\end{array}\right], \\
& \mathbb{D} z=\frac{12}{h^{2}}\left[\begin{array}{cccccccc}
\frac{115}{36} & \frac{-1555}{144} & \frac{89}{6} & \frac{-773}{72} & \frac{151}{36} & \frac{-11}{16} & \\
1 & -2 & 1 & & & & & \\
& 1 & -2 & 1 & & & & \\
& & & . & . & . & & \\
& & & & . & . & . & \\
& & & & 1 & -2 & 1 & \\
& & \frac{-11}{16} & \frac{151}{36} & \frac{-773}{72} & \frac{89}{6} & \frac{-1555}{144} & \frac{115}{36}
\end{array}\right]\left[\begin{array}{c}
z_{0} \\
z_{1} \\
z_{2} \\
z_{3} \\
\cdot \\
z_{N-3} \\
z_{N-2} \\
z_{N-1} \\
z_{N}
\end{array}\right] .
\end{aligned}
$$

\section{Implementation of CFDM6}

By substituting the values of first-order and second-order derivatives in Equations (3) and (8), a linear system of equations are obtained for $i=0,1, \cdots, N$ :

(i) Model-I: generalized Burger's-Fisher equation:

$$
\frac{\partial z_{i}}{\partial t}=\mathbb{C}^{-1} \mathbb{D} z_{i}-\beta z_{i}^{\delta} \mathbb{A}^{-1} \mathbb{B} z_{i}+\gamma z_{i}\left(1-z_{i}^{\delta}\right) \equiv \mathscr{L}\left(z_{i}\right) .
$$

(ii) Model-II: generalized Burger's-Huxley equation:

$$
\frac{\partial z_{i}}{\partial t}=\mathbb{C}^{-1} \mathbb{D} z_{i}-\beta z_{i}^{\delta} \mathbb{A}^{-1} \mathbb{B} z_{i}+\gamma z_{i}\left(1-z_{i}^{\delta}\right)\left(z_{i}^{\delta}-\eta\right) \equiv \mathscr{L}\left(z_{i}\right) .
$$




\subsection{SSP-RK43 Scheme. Let}

$$
\frac{d z_{i}}{d t}=\mathscr{L}\left(z_{i}\right), \quad i=0,1,2 \cdots, N
$$

where $\mathscr{L}$ represents the nonlinear differential operator as defined above. In order to solve this system of ODE's from the $t^{j}$ to $t^{j+1}$ time level, SSP-RK43 is applied using the following operations:

$$
\begin{aligned}
& \mathrm{z}^{(1)}=z^{j}+\frac{\Delta t}{2} \mathscr{L}\left(z^{j}\right), \\
& z^{(2)}=z^{(1)}+\frac{\Delta t}{2} \mathscr{L}\left(z^{(1)}\right), \\
& z^{(3)}=\frac{2}{3} z^{j}+\frac{1}{3} z^{(2)}+\frac{\Delta t}{6} \mathscr{L}\left(z^{(2)}\right), \\
& z^{j+1}=z^{(3)}+\frac{\Delta t}{2} \mathscr{L}\left(z^{(3)}\right) .
\end{aligned}
$$

By using the initial condition, $z(x, t)$ at every required time level can be calculated.

\section{Convergence Analysis}

Convergence of the model is investigated below for the desired Equations (22) and (23).

Theorem 1. It is an assumption that the given initial value problem $d z / d t=\mathscr{L}(z)$ has a unique solution if $\mathscr{L}(z)$ satisfies the following conditions:

(1) $\mathscr{L}(z)$ is a real function

(2) $\mathscr{L}(z)$ is well defined and continuous in the domain of $t \in \Phi_{t}$ and $z \in(-\infty, \infty)$

(3) There exists a constant called the Lipschitz constant $\kappa$ such that $|\mathscr{L}(z, t, \Delta t)-\mathscr{L}(\dot{z}, t, \Delta t)| \leq \kappa|z-\dot{z}|$, where $t \in \Phi_{t}$ and $z$ and $\dot{z}$ be any two different points

It is clearly seen that $\mathscr{L}(z)$ for the generalized Burger'sFisher equation and generalized Burger's-Huxley equation is real, well defined, and continuous. Hence, above theorem is satisfied.

Lemma 2. A single-step method (25) is said to be regular, if the incremental function $\phi(z, t, \Delta t)$ satisfies the following conditions:

(1) The function is well defined and is continuous in the given time and space domain

(2) For every $t \in \Phi_{t}$ and $z, \dot{z} \in(-\infty, \infty)$, there exit a constant $\kappa$ such that

$$
|\phi(z, t, \Delta t)-\phi(\dot{z}, t, \Delta t)| \leq \kappa|z-\dot{z}| .
$$

Lemma 3. Any single-step method is consistent if $\phi(z, t, 0)$ $=\mathscr{L}(z, t)$.

Theorem 4. The consistency is the necessary and sufficient condition for the convergence of a regular single-step method with the order (say) $p \geq 1$.

Proof. This theorem ensures that the approximate solution converges to the exact solution. For the proof, consider the specific incremental function $\phi(z, t, \Delta t)$. Assume that the given differential equation $z_{t} \equiv \mathscr{L} z$ has a unique solution $z(t)$ on $\Phi_{t}$ and also $z(t) \in C^{(p+1)} \Phi_{t}$ for $p \geq 1$. Using Taylor's series expansion about any point $t^{j}$,

$$
\begin{aligned}
z(t)= & z\left(t^{j}\right)+\left(t-t^{j}\right) z^{\prime}\left(t^{j}\right)+\frac{1}{2 !}\left(t-t^{j}\right)^{2} z^{\prime \prime}\left(t^{j}\right) \\
& +\cdots+\frac{1}{p !}\left(t-t^{j}\right)^{p} z^{p}\left(t^{j}\right)+\frac{1}{(p+1) !}\left(t-t^{j}\right)^{p+1} z^{p+1}\left(\xi^{j}\right),
\end{aligned}
$$

where $\xi \in\left(t^{j}, t\right)$. Taking $t=t^{j+1}$, one gets

$$
z\left(t^{j+1}\right)-z\left(t^{j}\right)=\Delta t z^{\prime}\left(t^{j}\right)
$$

Thus, the incremental function is defined as

$\phi\left(z\left(t^{j}\right), t^{j}, \Delta t\right)=(\Delta t) z^{\prime}\left(t^{j}\right)+\frac{1}{2 !}(\Delta t)^{2} z^{\prime \prime}\left(t^{j}\right)+\cdots+\frac{1}{p !}(\Delta t)^{p} z^{p}\left(t^{j}\right)$.

It is computed using the approximate value of $z^{j}$ where the exact value $z\left(t^{j}\right)$ is required. Hence, $z^{j+1}=z^{j}+$ $\Delta t \phi\left(z\left(t^{j}\right), t^{j}, \Delta t\right), j=0,1,2, \cdots, m-1$. To compute the error using Taylor's series,

$$
\begin{aligned}
z^{j+1}= & z^{j}+\Delta t z^{\prime j}+\frac{(\Delta t)^{2}}{2 !} z^{\prime \prime j}+\frac{(\Delta t)^{3}}{3 !} z^{\prime \prime \prime j} \\
& +\cdots+\frac{(\Delta t)^{p}}{p !} z^{p j}+\frac{(\Delta t)^{p+1}}{(p+1) !} z^{p+1}(\xi)^{j} .
\end{aligned}
$$

The approximate value using the SSP-RK43 scheme is

$$
\begin{aligned}
z^{j+1}= & z^{j}+\Delta t \mathscr{L}\left(z^{j}\right)+\frac{(\Delta t)^{2}}{2 !} \mathscr{L}^{2}\left(z^{j}\right)+\frac{(\Delta t)^{3}}{3 !} \mathscr{L}^{3}\left(z^{j}\right) \\
& +\cdots+\frac{(\Delta t)^{p}}{p !} \mathscr{L}^{p}\left(z^{j}\right) .
\end{aligned}
$$

The following relation is obtained:

$$
\begin{aligned}
\Delta t \phi\left(z\left(t^{j}\right), t^{j}, \Delta t\right)= & \Delta t z^{\prime}\left(t^{j}\right)+\frac{(\Delta t)^{2}}{2 !} \mathscr{L}^{2}\left(z^{j}\right)+\frac{(\Delta t)^{3}}{3 !} \mathscr{L}^{3}\left(z^{j}\right) \\
& +\cdots+\frac{(\Delta t)^{p}}{p !} \mathscr{L}^{p}\left(z^{j}\right) .
\end{aligned}
$$




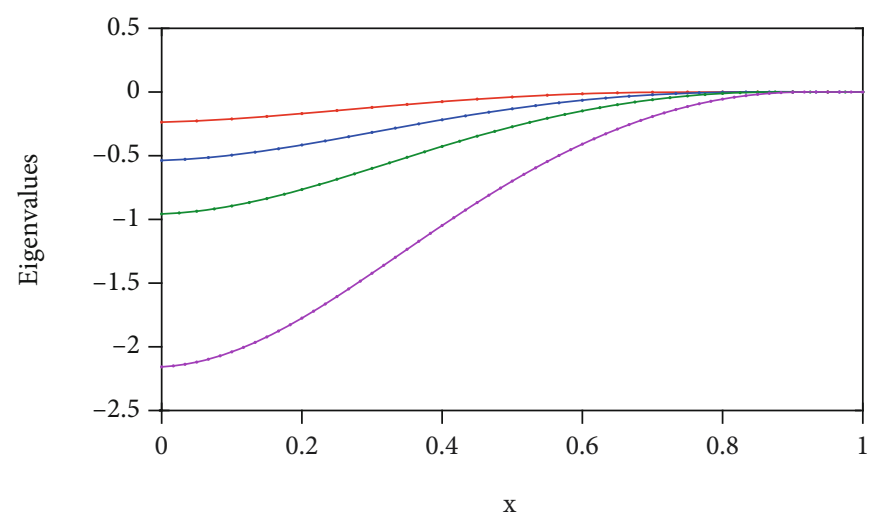

(a) $\beta=0.001, \gamma=0.001$

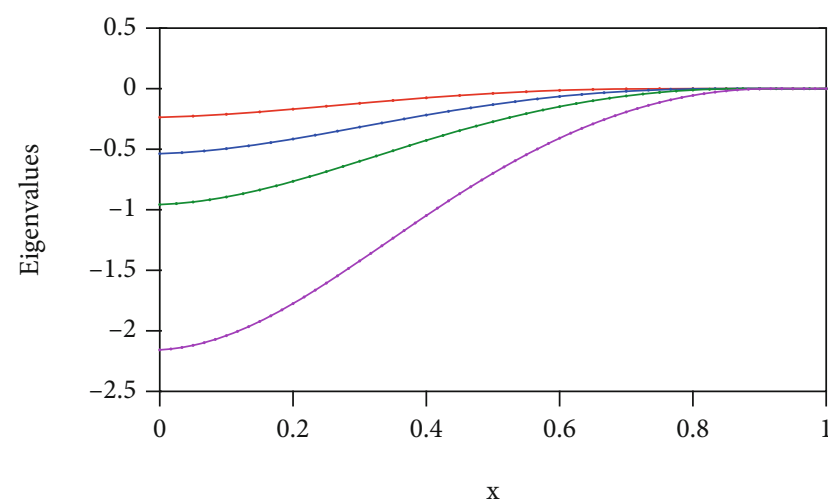

(b) $\beta=1, \gamma=1$

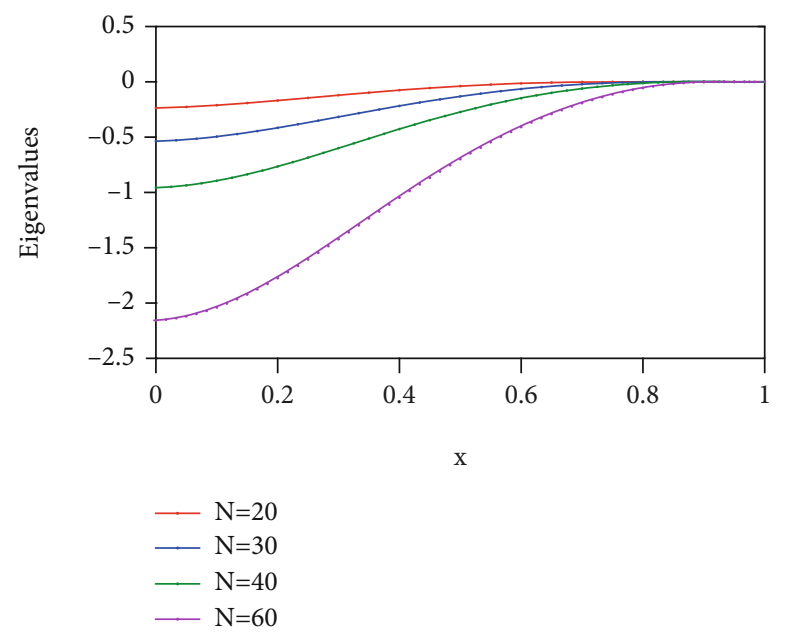

(c) $\beta=0.1, \gamma=-0.0025$

Figure 1: Plot of eigenvalues corresponding to gBF equation with $\Delta t=0.0001$ and $\delta=8$.

The value of $\Delta t \phi\left(z^{j}, t^{j}, \Delta t\right)$ is obtained from $\Delta t \phi\left(z\left(t^{j}\right)\right.$, $t^{j}, \Delta t$ ) by using the exact approximate value of $z^{j}$ in place of the exact value of $z\left(t^{j}\right)$. According to the SSP-RK43, the approximate value of $z\left(t^{j+1}\right)$ is obtained as follows:

$z^{j+1}=z^{j}+\Delta t \phi\left(z^{j}, t^{j}, \Delta t\right)+\frac{(\Delta t)^{2}}{2 !} \phi^{\prime}\left(z^{j}, t^{j}, \Delta t\right)+\frac{(\Delta t)^{3}}{3 !} \phi^{\prime \prime}\left(z^{j}, t^{j}, \Delta t\right)+\cdots$

For the above relation, compute the values of $z\left(t^{j}\right), z^{\prime}\left(t^{j}\right)$, $z^{\prime \prime}\left(t^{j}\right) \cdots z^{p}\left(t^{j}\right)$ as follows:

$$
\begin{gathered}
z^{\prime}\left(t^{j}\right)=\mathscr{L}\left(z\left(t^{j}\right), t^{j}\right), \\
z^{\prime \prime}\left(t^{j}\right)=\mathscr{L}_{t}+\mathscr{L}_{z}, \\
z^{\prime \prime \prime}\left(t^{j}\right)=\mathscr{L}_{t t}+2^{\prime} \mathscr{L}_{t z}+\mathscr{L}^{2} L_{z z}+L_{z}\left(\mathscr{L}_{t}+\mathscr{L}_{z}\right) .
\end{gathered}
$$

Thus, from these computed values taking $t=t^{j}$, the error term is obtained as follows:

$$
\frac{\Delta t^{p+1}}{(p+1) !} z^{p+1}\left(\xi^{j}\right)<\varepsilon
$$

Hence, on simplification,

$$
\Delta t^{p+1} z^{p+1}\left(\xi^{j}\right)<\varepsilon(p+1) !
$$

In other words,

$$
\Delta t^{p+1} \mathscr{L}^{p}\left(\xi^{j}\right)<\varepsilon(p+1) !
$$

Thus, the given value of $p$ will give the upper bound, and for the computational purpose, the value of $\mathscr{L}^{p}\left(\xi^{j}\right)$ in Equation (37) is replaced with the $\max \left|\mathscr{L}^{p}\left(\xi^{j}\right)\right|$ in the temporal domain $\Phi_{t}$. The SSP-RK43 as discussed above is rewritten as 


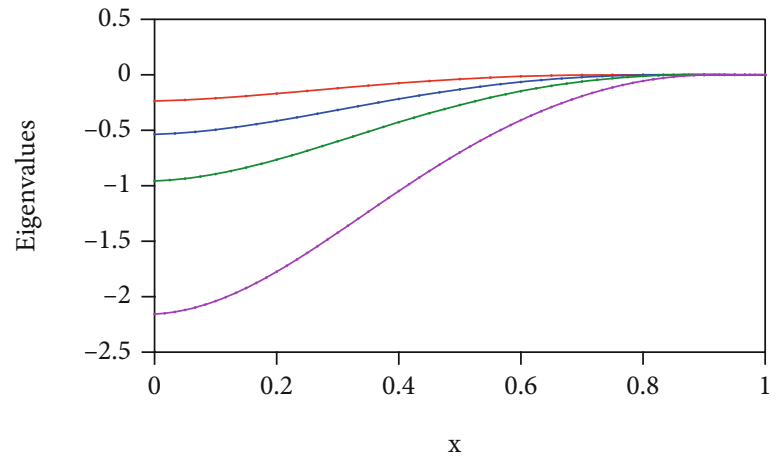

(a) $\beta=\gamma=1, \eta=0.001$

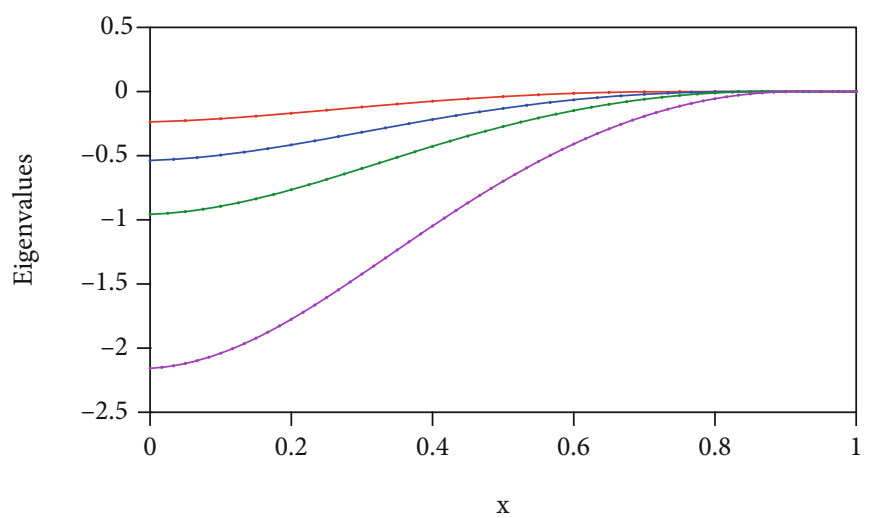

(c) $\beta=5 \gamma=10, \eta=0.0001$

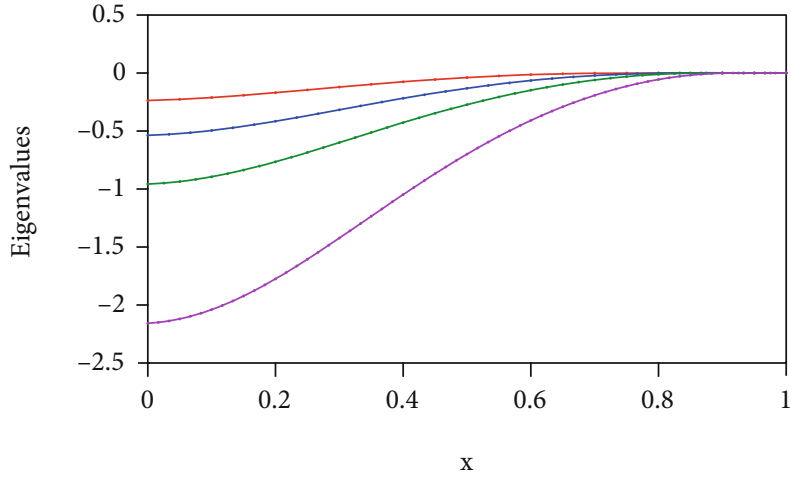

(b) $\beta=0.1, \gamma=0.001, \eta=0.0001$
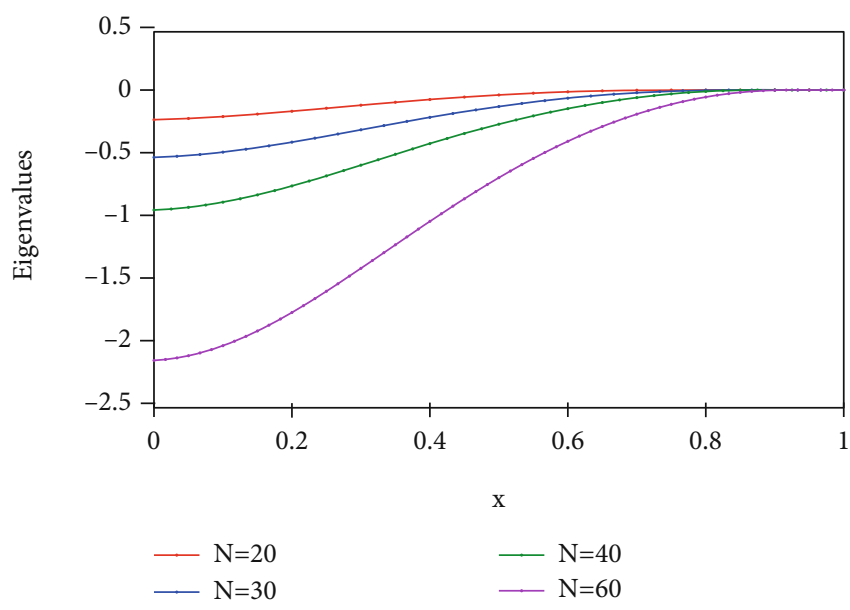

(d) $\beta=0 \gamma=1, \eta=0.001$

Figure 2: Plot of eigenvalues corresponding to gBH equation with $\Delta t=0.0001$ and $\delta=8$.

$$
\begin{aligned}
Q_{1} & =z^{j}+\frac{\Delta t}{2} \mathscr{L}\left(z^{j}, t^{j}\right), \\
Q_{2} & =Q_{1}+\frac{\Delta t}{2} \mathscr{L}\left(Q_{1}\right), \\
Q_{3} & =\frac{2}{3} z^{j}+\frac{1}{3} Q_{2}+\frac{\Delta t}{6} \mathscr{L}\left(Q_{2}\right), \\
z^{j+1} & =Q_{3}+\frac{\Delta t}{2} \mathscr{L}\left(Q_{3}\right) .
\end{aligned}
$$

The iterated value of $z^{j+1}$ can be written as

$$
z^{j+1}=z^{j}+c_{1} Q_{1}+c_{2} Q_{2}+c_{3} Q_{3}
$$

Using Taylor's series expansion, the incremental function becomes

$$
\phi\left(z^{j}, t^{j}, \Delta t\right)=(\Delta t)^{-1}\left(c_{1} Q_{1}+c_{2} Q_{2}+c_{3} Q_{3}\right)
$$

From the Theorem 1, the proof for convergence is elaborated as follows:

$$
Q_{1}-Q_{1}{ }^{*}=z^{j}+\frac{\Delta t}{2} \mathscr{L}\left(z^{j}\right)-z^{j^{*}}+\frac{\Delta t}{2} \mathscr{L}\left(z^{j^{*}}\right)
$$

$$
\begin{aligned}
& \left|Q_{1}-Q_{1}{ }^{*}\right| \leq\left|z^{j}-z^{j^{*}}\right|+\frac{\Delta t}{2}\left|\mathscr{L}\left(z^{j}\right)-\mathscr{L}\left(z^{j^{*}}\right)\right| \leq\left(1+\frac{\Delta t}{2} \kappa\right)\left|z^{j}-z^{j^{*}}\right|, \\
& Q_{2}-Q_{2}{ }^{*}=Q_{1}+\frac{\Delta t}{2} \mathscr{L}\left(Q_{1}\right)-Q_{1}{ }^{*}-\frac{\Delta t}{2} \mathscr{L}\left(Q_{1}{ }^{*}\right) \\
& \left|Q_{2}-Q_{2}{ }^{*}\right| \leq\left|Q_{1}-Q_{1}{ }^{*}\right|+\frac{\Delta t}{2}\left|\mathscr{L}\left(Q_{1}\right)-\mathscr{L}\left(Q_{1}{ }^{*}\right)\right| \\
& =\left|Q_{1}-Q_{1}{ }^{*}\right|+\frac{\Delta t}{2}\left|\mathscr{L}\left(z^{j}+\frac{\Delta t}{2} \mathscr{L}\left(z^{j}\right)\right)-\mathscr{L}\left(z^{j^{*}}+\frac{\Delta t}{2} \mathscr{L}\left(z^{j^{*}}\right)\right)\right| \\
& \leq\left(1+\frac{\Delta t}{2} \kappa\right)\left|z^{j}-z^{j^{*}}\right| \\
& +\frac{\Delta t}{2}\left[\mathscr{L}\left(z^{j}\right)+\frac{\Delta t}{2} \mathscr{L}\left(z^{j}\right) \mathscr{L}_{z}\left(z^{j}\right)+\left(\frac{\Delta t}{2} \mathscr{L}\left(z^{j}\right)\right)^{2} \mathscr{L}_{z z}\left(z^{j}\right)\right. \\
& \left.+\cdots-\mathscr{L}\left(z^{j^{*}}\right)-\frac{\Delta t}{2} \mathscr{L}\left(z^{j^{*}}\right) \mathscr{L}_{z}\left(z^{j^{*}}\right)-\left(\frac{\Delta t}{2} \mathscr{L}\left(z^{j^{*}}\right)\right)^{2} \mathscr{L}_{z z}\left(z^{j^{*}}\right)\right] \\
& \leq\left(1+\frac{\Delta t}{2} \kappa\right)\left|z^{j}-z^{j^{*}}\right|+\frac{\Delta t}{2}\left[\mathscr{L}\left(z^{j}\right)-\mathscr{L}\left(z^{j^{*}}\right)\right] \\
& +\left(\frac{\Delta t}{2}\right)^{2}\left|\mathscr{L}\left(z^{j}\right) \mathscr{L}_{z}\left(z^{j}\right)-\mathscr{L}\left(z^{j^{*}}\right) \mathscr{L}_{z}\left(z^{j^{*}}\right)\right| \\
& +\left(\frac{\Delta t}{2}\right)^{3}\left|\left(\mathscr{L}\left(z^{j}\right)\right)^{2} \mathscr{L}_{z z}\left(z^{j}\right)-\left(\mathscr{L}\left(z^{j^{*}}\right)\right)^{2} \mathscr{L}_{z z}\left(z^{j^{*}}\right)\right|+\cdots \\
& \leq(1+\Delta t \kappa)\left|z^{j}-z^{j^{*}}\right|+\left(\frac{\Delta t}{2}\right)^{2} \kappa^{2}\left|z^{j}-z^{j^{*}}\right| \\
& =\left[1+\Delta t \kappa+\left(\frac{\Delta t}{2} \kappa\right)^{2}\right]\left|z^{j}-z^{j^{*}}\right|,
\end{aligned}
$$


TABLE 1: Comparison of absolute error of Example 1 with $\beta=0.001, \gamma=0.001, h=0.1$, and $\Delta t=0.0001$.

\begin{tabular}{|c|c|c|c|c|c|c|c|c|}
\hline \multirow[b]{2}{*}{$t$} & \multirow[b]{2}{*}{$x$} & \multicolumn{4}{|c|}{$\delta=1$} & \multicolumn{3}{|c|}{$\delta=4$} \\
\hline & & CFDM6 & $\mathrm{ADM}[23]$ & CFDM [6] & ETDM [14] & CFDM6 & CFDM [6] & ETDM [14] \\
\hline \multirow{3}{*}{0.001} & 0.1 & $2.2204 E-16$ & $1.94 E-06$ & $1.01 E-07$ & $1.15 E-08$ & $1.1102 E-16$ & $1.75 E-08$ & $7.71 E-09$ \\
\hline & 0.5 & $1.1102 E-16$ & $1.94 E-06$ & $1.04 E-07$ & $3.07 E-13$ & $1.1102 E-16$ & $1.75 E-08$ & $2.07 E-13$ \\
\hline & 0.9 & $4.4409 E-17$ & $1.94 E-06$ & $1.01 E-07$ & $1.15 E-08$ & $3.3307 E-16$ & $1.75 E-08$ & $7.71 E-09$ \\
\hline \multirow{3}{*}{0.010} & 0.1 & $5.8818 E-16$ & $1.94 E-05$ & $7.53 E-07$ & $6.02 E-08$ & $4.4409 E-15$ & $1.27 E-06$ & $4.05 E-08$ \\
\hline & 0.5 & $1.6653 E-16$ & $1.94 E-05$ & $1.04 E-06$ & $8.96 E-13$ & $4.2188 E-15$ & $1.75 E-06$ & $5.56 E-13$ \\
\hline & 0.9 & $1.1102 E-15$ & $1.94 E-05$ & $7.53 E-07$ & $6.02 E-08$ & $4.8850 E-15$ & $1.27 E-06$ & $4.05 E-08$ \\
\hline \multirow{3}{*}{100} & 0.1 & $2.2204 E-16$ & - & $7.53 E-07$ & $1.01 E-07$ & $5.5511 E-16$ & - & $5.73 E-08$ \\
\hline & 0.5 & $1.1102 E-15$ & - & $1.04 E-06$ & $1.50 E-11$ & $2.7756 E-15$ & - & $3.51 E-12$ \\
\hline & 0.9 & $1.1102 E-16$ & - & $7.53 E-07$ & $1.01 E-07$ & $1.3323 E-15$ & - & $5.73 E-08$ \\
\hline
\end{tabular}

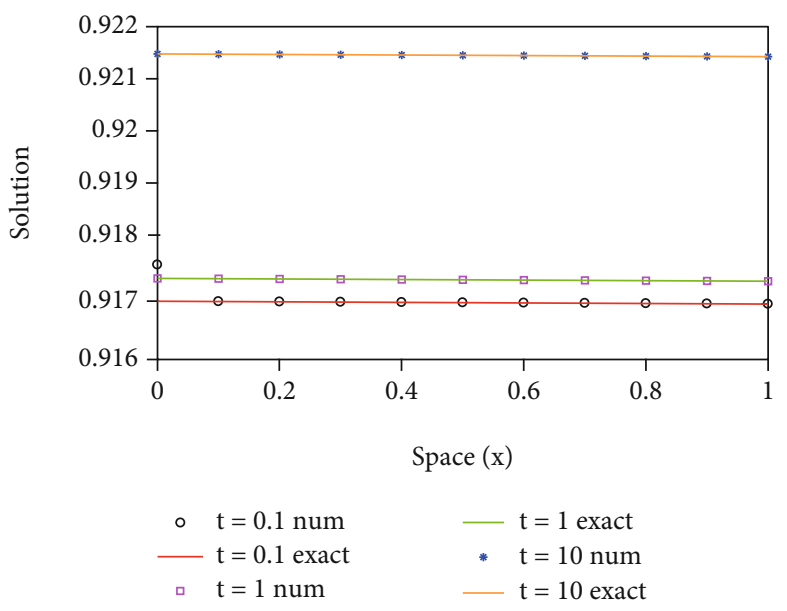

(a) Comparison of numerical and exact solution

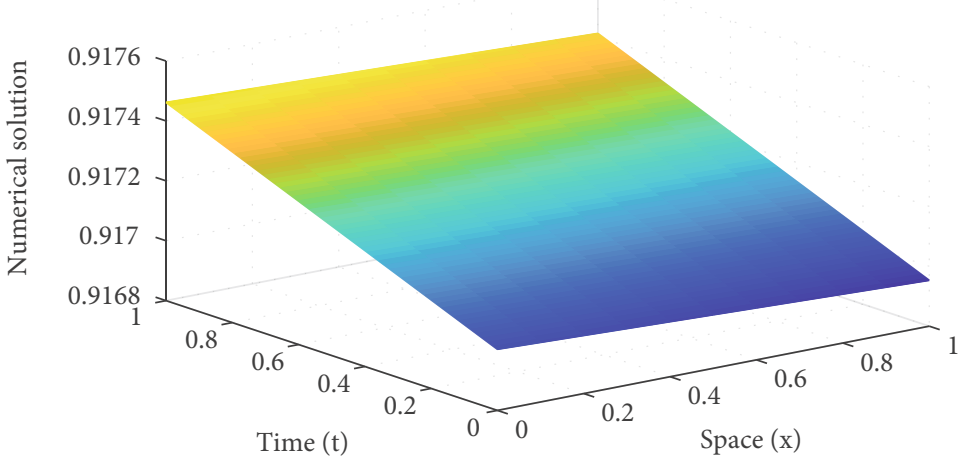

(b) Surface plot of numerical solution

Figure 3: Graphical representation of solutions corresponding to Example 1 with $N=10$ and $\Delta t=0.001$.

$\left.Q_{3}-Q_{3}{ }^{*}=\frac{2}{3} z^{j}+\frac{Q_{2}}{3}+\frac{\Delta t}{2} \mathscr{L}\left(Q_{2}\right)-\frac{2}{3} z^{j *}-\frac{Q_{2}{ }^{*}}{3}-\frac{\Delta t}{2} \mathscr{L}\left(Q_{2}{ }^{*}\right)\right)$,

$$
\begin{aligned}
\left|Q_{3}-Q_{3}{ }^{*}\right|= & \frac{2}{3}\left|z^{j}-z^{j^{*}}\right|+\frac{1}{3}\left|Q_{2}-Q_{2}{ }^{*}\right|+\frac{\Delta t}{2}\left|\mathscr{L}\left(Q_{2}\right)-\mathscr{L}\left(Q_{2}{ }^{*}\right)\right| \\
\leq & \frac{2}{3}\left|z^{j}-z^{j^{*}}\right|+\frac{1}{3}\left|Q_{2}-Q_{2}{ }^{*}\right| \\
& +\left|\frac{\Delta t}{2}\left[\mathscr{L}\left(Q_{1}+\frac{\Delta t}{2} \mathscr{L}\left(Q_{1}\right)\right)-\mathscr{L}\left(Q_{1}^{*}+\frac{\Delta t}{2} \mathscr{L}\left(Q_{1}^{*}\right)\right)\right]\right| \\
\leq & \frac{2}{3}\left|z^{j}-z^{j^{*}}\right|+\frac{1}{3}\left|Q_{2}-Q_{2}{ }^{*}\right| \\
& +\frac{\Delta t}{2}\left[\left|\left(\mathscr{L}\left(z^{j}\right)-\mathscr{L}\left(z^{j^{*}}\right)\right)\right|+\frac{\Delta t}{2}\left|\mathscr{L}\left(z^{j}\right) \mathscr{L}_{z}\left(z^{j}\right)-\mathscr{L}_{z}\left(z^{j^{*}}\right)\right|\right] \\
\leq & \left|z^{j}-z^{j^{*}}\right|+\frac{\Delta t}{2} \kappa\left(2+\frac{\Delta t}{2} \kappa\right)\left|z^{j}-z^{j^{*}}\right| \\
& +\frac{\Delta t}{2} \kappa\left|z^{j}-z^{j^{*}}\right|+\left(\frac{\Delta t}{2} \kappa\right)\left|z^{j}-z^{j^{*}}\right| \\
\leq & \left|z^{j}-z^{j^{*}}\right|+\left[\frac{3 \Delta t}{2} \kappa+2\left(\frac{\Delta t}{2} \kappa\right)^{2}\right]\left|z^{j}-z^{j^{*}}\right|,
\end{aligned}
$$

As discussed by [47], the free parameters are largely taken according to the range of absolute stability. The other possibility is minimizing the sum of the absolute value of the coefficients of the truncation error. Thus $\mathscr{L}_{z}<\kappa$ and $\mathscr{L}_{z z}<\kappa^{2} / M$ where $M$ is the upper bound of convergence. For the incremental function,

$$
\begin{aligned}
\mid \phi & \left(z^{j}, t^{j}, \Delta t\right)-\phi\left(z^{j^{*}}, t^{j}, \Delta t\right) \mid \\
= & (\Delta t)^{-1}\left|c_{1} Q_{1}+c_{2} Q_{2}+c_{3} Q_{3}-c_{1} Q_{1}{ }^{*}-c_{2} Q_{2}{ }^{*}-c_{3} Q_{3}{ }^{*}\right| \\
= & (\Delta t)^{-1}\left(c_{1}\left|Q_{1}-Q_{1}{ }^{*}\right|+c_{2}\left|Q_{2}-Q_{2}{ }^{*}\right|+c_{3}\left|Q_{3}-Q_{3}{ }^{*}\right|\right) \\
\leq & (\Delta t)^{-1}\left[c_{1}\left(1+\frac{\Delta t}{2} \kappa\right)\left|z^{j}-z^{j^{*}}\right|+c_{2}\left(\left[1+\Delta t \kappa+\left(\frac{\Delta t}{2} \kappa\right)^{2}\right]\left|z^{j}-z^{j^{*}}\right|\right)\right. \\
& \left.+c_{3}\left(\left|z^{j}-z^{j^{*}}\right|+\left[\frac{3 \Delta t}{2} \kappa+2\left(\frac{\Delta t}{2} \kappa\right)^{2}\right]\left|z^{j}-z^{j^{*}}\right|\right)\right] \\
\leq & {\left[(\Delta t)^{-1}\left(c_{1}+c_{2}+c_{3}\right)+\left[c_{1}+2 c_{2}+c_{3}\right] \frac{\kappa}{2}+\left[c_{2}+2 c_{3}\right] \Delta t\left(\frac{\kappa}{2}\right)^{2}\right]\left|z^{j}-z^{j^{*}}\right| . }
\end{aligned}
$$

The backward substitution of (38) and its comparison with general Taylor's series [47] gives $c_{1}=1 / 4, c_{2}=1 / 2, c_{3}=1 / 4$. Hence, these values generate the inequality as

$$
\left|\phi\left(z^{j}, t^{j}, \Delta t\right)-\phi\left(z^{j^{*}}, t^{j}, \Delta t\right)\right| \leq \kappa\left(1+\frac{1}{2} \Delta t \kappa+\frac{1}{6}(\Delta t \kappa)^{2}\right)\left|z^{j}-z^{j^{*}}\right| .
$$


TABLE 2: Comparison of absolute error of Example 2 with $\beta=1, \gamma=1, h=0.1$, and $\Delta t=0.0001$.

\begin{tabular}{|c|c|c|c|c|c|c|c|c|}
\hline \multirow[b]{2}{*}{$t$} & \multirow[b]{2}{*}{$x$} & \multicolumn{4}{|c|}{$\delta=2$} & \multicolumn{3}{|c|}{$\delta=8$} \\
\hline & & CFDM6 & $\mathrm{ADM}$ [23] & CFDM [6] & ETDM [14] & CFDM6 & CFDM [6] & ETDM [14] \\
\hline \multirow{3}{*}{0.0005} & 0.1 & $2.2547 E-11$ & $1.40 E-03$ & $7.62 E-05$ & $5.67 E-06$ & $4.8073 E-11$ & $1.02 E-04$ & $2.44 E-06$ \\
\hline & 0.5 & $8.4710 E-14$ & $1.35 E-03$ & $9.14 E-05$ & $5.75 E-09$ & $1.6162 E-12$ & $1.37 E-04$ & $1.82 E-10$ \\
\hline & 0.9 & $1.8019 E-11$ & $1.28 E-03$ & $1.02 E-04$ & $5.95 E-06$ & $6.3383 E-13$ & $1.69 E-04$ & $3.15 E-06$ \\
\hline \multirow{3}{*}{0.0010} & 0.1 & $4.3846 E-11$ & $2.80 E-03$ & $1.50 E-04$ & $1.08 E-05$ & $9.3434 E-11$ & $2.00 E-04$ & $4.65 E-06$ \\
\hline & 0.5 & $1.8086 E-13$ & $2.69 E-03$ & $1.83 E-04$ & $1.15 E-08$ & $3.2596 E-12$ & $2.74 E-04$ & $4.02 E-10$ \\
\hline & 0.9 & $3.5862 E-12$ & $2.55 E-03$ & $2.00 E-04$ & $1.14 E-05$ & $1.6023 E-12$ & $3.31 E-04$ & $6.00 E-06$ \\
\hline
\end{tabular}

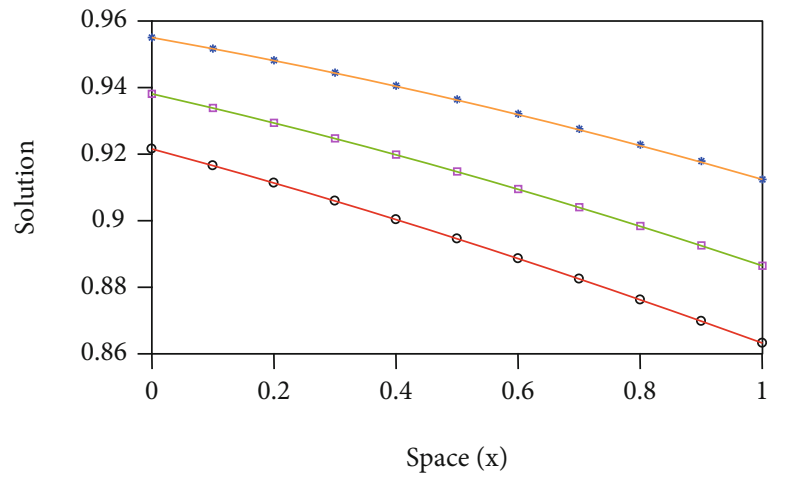

$$
\begin{aligned}
& \text { - } \mathrm{t}=0.1 \text { num } \quad \mathrm{t}=0.5 \text { exact } \\
& -\mathrm{t}=0.1 \text { exact } \quad * \mathrm{t}=1 \text { num } \\
& \text { 口 } \mathrm{t}=0.5 \text { num } \quad \mathrm{t}=1 \text { exact }
\end{aligned}
$$

(a) Comparison of numerical and exact solution

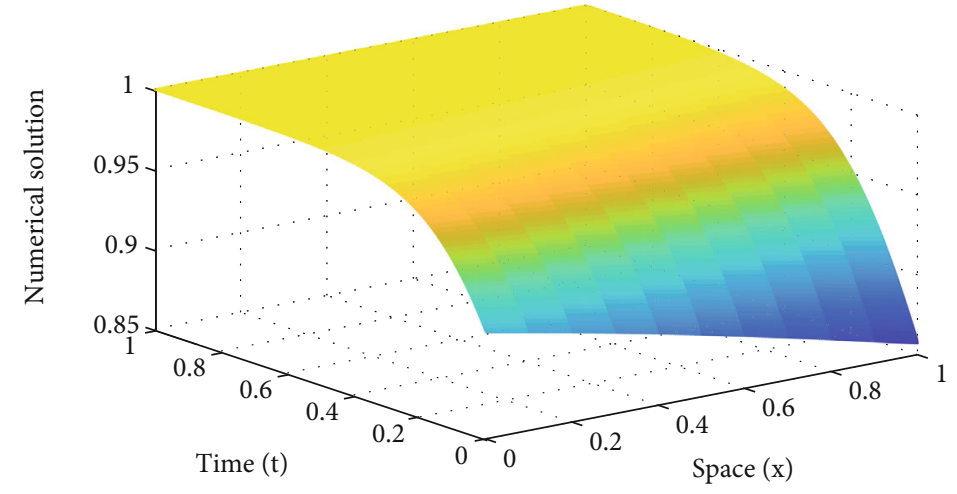

(b) Surface plot of numerical solution

FIGURE 4: Graphical representation of solutions corresponding to Example 2 with $N=10$ and $\Delta t=0.001$.

It is observed that $\left|\phi\left(z^{j}, t^{j}, \Delta t\right)\right|$ satisfies the Lipschitz condition in $z^{j}$ and is a continuous function in $\Delta t$. Thus, it is concluded that SSP-RK43 is convergent.

$z_{t}=\left(\mathbb{C}^{-1} \mathbb{D}-\left(\beta m^{\delta} \mathbb{A}^{-1} \mathbb{B} z_{i}+\gamma\left(1-m^{\delta}\right)\left(m^{\delta}-\eta\right)\right) I\right) z_{i} \equiv \mathfrak{I} z_{i}, \frac{d z}{d t}=\mathfrak{T} z$

\section{Stability Analysis}

The stability analysis of both the models is discussed below by taking nonlinearity coefficient $z=m$ (say), where $m=$ $\max z$, in the entire process to handle the nonlinear term in Equations (22) and (23). The eigenvalue-based technique [45] is followed to establish the stability of the system.

(1) Model-I: generalized Burger's-Fisher equation:

$$
\begin{aligned}
\frac{\partial z}{\partial t} & =\mathbb{C}^{-1} \mathbb{D} z_{i}-\beta m^{\delta} \mathbb{A}^{-1} \mathbb{B} z_{i}+\gamma\left(1-m^{\delta}\right) z_{i}, \\
z_{t} & =\left(\mathbb{C}^{-1} \mathbb{D}-\left(\beta m^{\delta} \mathbb{A}^{-1} \mathbb{B}+\gamma\left(1-m^{\delta}\right)\right) I\right) z_{i} \equiv \mathfrak{I} z_{i} .
\end{aligned}
$$

(2) Model-II: generalized Burger's-Huxley equation:

$$
\frac{\partial z_{i}}{\partial t}=\mathbb{C}^{-1} \mathbb{D} z_{i}-\beta m^{\delta} \mathbb{A}^{-1} \mathbb{B} z_{i}+\gamma\left(1-m^{\delta}\right)\left(m^{\delta}-\eta\right) z_{i}
$$

The matrix $\mathfrak{I}$ is constant for both the Model-I and Model-II with the assumption that it has distinct or possibly complex eigenvalues with a negative real part. Using the given initial condition for the analytic solution, the relation becomes

$$
z(t)=\exp (\mathfrak{I} t) z^{0}
$$

whereas on expanding the exponent as a matrix function where $I$ is the identity matrix,

$$
\exp (\mathfrak{T} t)=I+\mathfrak{T} t+\frac{(\mathfrak{T} t)^{2}}{2 !}+\frac{(\mathfrak{T} t)^{3}}{3 !}+\cdots
$$

For Model-I and Model-II, consider the transformation matrix $P$ such that $P^{-1} \mathfrak{T} P=\mathscr{D}$ where $\mathscr{D}$ is the diagonal matrix; thus, the relation becomes

$$
P^{-1} \exp (\mathfrak{I} t) P=\exp (\mathscr{D} t)
$$


TABLE 3: Comparison of absolute error of Example 3 with $\beta=0.1, \gamma=-0.0025, h=0.1$, and $\Delta t=0.0001$.

\begin{tabular}{|c|c|c|c|c|c|c|c|c|c|c|}
\hline$t$ & $x$ & CFDM6 & $\begin{array}{c}\delta=2 \\
\text { CFDM [6] }\end{array}$ & ETDM [14] & CFDM6 & $\begin{array}{c}\delta=4 \\
\text { CFDM [6] }\end{array}$ & ETDM [14] & CFDM6 & $\begin{array}{c}\delta=8 \\
\text { CFDM [6] }\end{array}$ & ETDM [14] \\
\hline \multirow{3}{*}{0.1} & 0.1 & $6.661 E-16$ & $1.21 E-05$ & $9.47 E-06$ & $2.220 E-16$ & $1.34 E-05$ & $6.76 E-06$ & $1.110 E-15$ & $1.47 E-05$ & $4.09 E-06$ \\
\hline & 0.5 & $6.661 E-16$ & $2.90 E-05$ & $2.74 E-08$ & $5.551 E-16$ & $3.49 E-05$ & $1.03 E-08$ & $6.661 E-16$ & $3.83 E-05$ & $1.84 E-08$ \\
\hline & 0.9 & $2.220 E-16$ & $1.54 E-05$ & $9.57 E-06$ & $7.772 E-16$ & $1.39 E-05$ & $6.92 E-08$ & $3.331 E-16$ & $1.53 E-05$ & $4.24 E-06$ \\
\hline \multirow{3}{*}{0.5} & 0.1 & $1.341 E-16$ & $1.67 E-05$ & $9.58 E-06$ & $6.661 E-16$ & $2.00 E-05$ & $6.83 E-06$ & $4.441 E-16$ & $2.20 E-05$ & $4.14 E-06$ \\
\hline & 0.5 & $1.887 E-15$ & $4.69 E-05$ & $5.18 E-08$ & $2.331 E-15$ & $5.64 E-05$ & $1.93 E-08$ & $6.661 E-16$ & $6.22 E-05$ & $3.47 E-08$ \\
\hline & 0.9 & $4.441 E-16$ & $1.71 E-05$ & $9.66 E-06$ & $1.665 E-15$ & $2.07 E-05$ & $7.01 E-06$ & $1.332 E-15$ & $2.28 E-05$ & $4.30 E-06$ \\
\hline \multirow{3}{*}{2.0} & 0.1 & $5.551 E-16$ & - & $9.59 E-06$ & $1.221 E-15$ & - & $6.86 E-06$ & $1.221 E-15$ & - & $4.20 E-06$ \\
\hline & 0.5 & $3.331 E-15$ & - & $5.26 E-08$ & $1.776 E-15$ & - & $1.89 E-08$ & $3.997 E-15$ & - & $3.45 E-08$ \\
\hline & 0.9 & $6.661 E-16$ & - & $9.67 E-06$ & $7.772 E-16$ & - & $7.04 E-06$ & $3.331 E-16$ & - & $4.35 E-06$ \\
\hline
\end{tabular}

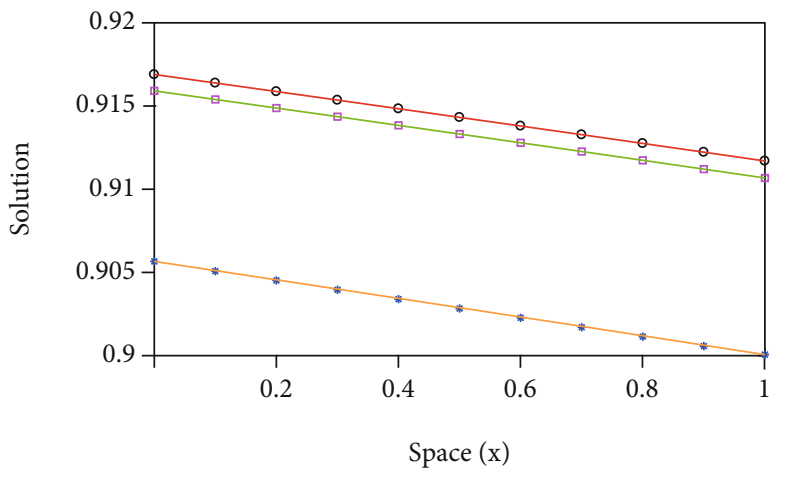

$$
\begin{aligned}
\mathrm{t} & =0.1 \text { num } \\
\mathrm{t} & =0.1 \text { exact } \\
\mathrm{t} & =1 \text { num }
\end{aligned} \quad \begin{aligned}
\mathrm{t} & =1 \text { exact } \\
\mathrm{t} & =10 \text { num } \\
\mathrm{t} & =10 \text { exact }
\end{aligned}
$$

(a) Comparison of numerical and exact solution

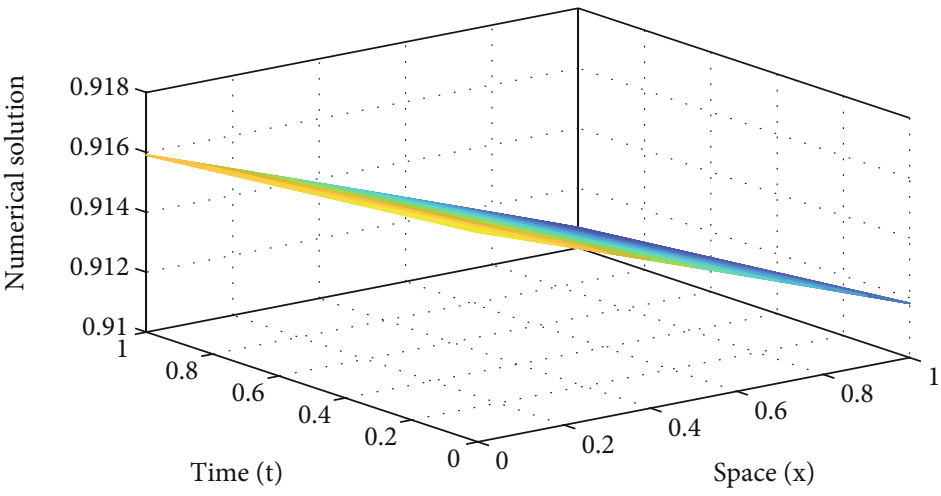

(b) Surface plot of numerical solution

FIGURE 5: Graphical representation of solutions corresponding to Example 3 with $N=10$ and $\Delta t=0.001$.

TABLE 4: Comparison of absolute error of Example 4 with $\beta=1, \gamma=1, \eta=0.001, \delta=2, h=0.1$, and $\Delta t=0.0001$.

\begin{tabular}{|c|c|c|c|c|c|c|}
\hline \multirow{2}{*}{ Method } & \multicolumn{3}{|c|}{$t=0.2$} & \multicolumn{3}{|c|}{$t=1$} \\
\hline & $\delta=1$ & $\delta=4$ & $\delta=8$ & $\delta=1$ & $\delta=4$ & $\delta=8$ \\
\hline CFDM6 $(\Delta t=0.1)$ & $7.4965 E-08$ & $1.3207 E-07$ & $1.3587 E-07$ & $3.7494 E-07$ & $6.6011 E-07$ & $6.7896 E-07$ \\
\hline MCSCM [51] & $3.7487 E-08$ & $1.2271 E-05$ & $3.3191 E-05$ & $4.2940 E-08$ & $1.4046 E-05$ & $3.7949 E-05$ \\
\hline MGT [52] & $4.0305 E-08$ & $1.3193 E-05$ & $3.5687 E-05$ & $4.6849 E-08$ & $1.5325 E-05$ & $4.1407 E-05$ \\
\hline UAHT [37] & $1.8104 E-08$ & $5.9274 E-06$ & $1.6034 E-05$ & $1.8219 E-08$ & $5.9602 E-06$ & $1.6102 E-05$ \\
\hline UAH [37] & $4.0069 E-08$ & $1.3118 E-05$ & $3.5485 E-05$ & $4.6833 E-08$ & $1.5321 E-05$ & $4.1400 E-05$ \\
\hline UAT [37] & $4.0326 E-08$ & $1.3202 E-05$ & $3.5712 E-05$ & $4.6834 E-08$ & $1.5322 E-05$ & $4.1400 E-05$ \\
\hline
\end{tabular}

\begin{tabular}{lcccccc}
\hline Method & & & & \multicolumn{1}{c}{$t=1$} \\
$x=0.1$ & $x=0.9$ & $x=0.1$ & $x=0.9$ \\
\hline CFDM6 $(\Delta t=0.1)$ & $6.4123 E-08$ & $6.4126 E-08$ & $6.4129 E-08$ & $6.4099 E-07$ & $6.4102 E-07$ & $6.4105 E-07$ \\
EFD [49] & $2.0510 E-06$ & $5.2339 E-06$ & $2.0511 E-06$ & $3.0562 E-06$ & $8.4901 E-06$ & $3.0564 E-06$ \\
HSCM [50] & $5.1820 E-07$ & $1.3220 E-06$ & $5.1820 E-07$ & $7.7340 E-07$ & $2.1480 E-06$ & $7.7340 E-07$ \\
UAHT [37] & $2.8510 E-07$ & $7.8223 E-07$ & $2.8507 E-07$ & $3.0616 E-07$ & $8.5042 E-07$ & $3.0614 E-07$ \\
UAH [37] & $5.2629 E-07$ & $1.3423 E-06$ & $5.2620 E-07$ & $7.8705 E-07$ & $2.1860 E-06$ & $7.8690 E-07$ \\
UAT [37] & $5.3131 E-07$ & $1.3585 E-06$ & $5.3121 E-07$ & $7.8706 E-07$ & $2.1861 E-06$ & $7.8691 E-07$ \\
\hline
\end{tabular}

TABLE 5: Comparison of $L_{\infty}$ error norm of Example 4 with $\beta=1, \gamma=1, \eta=0.001, h=0.1$, and $\Delta t=0.001$. 
TABLE 6: Comparison of error norms of Example 4 with $\beta=1, \gamma=1, \eta=0.001, \delta=2, h=0.1$, and $\Delta t=0.01$.

\begin{tabular}{lcccrr}
\hline Method & Error (time) & $t=0.05$ & $t=0.1$ & $t=1$ & \multicolumn{1}{c}{$t=5$} \\
\hline \multirow{2}{*}{ CFDM6 } & $L_{\infty}$ & $3.2065 E-08$ & $6.4129 E-08$ & $6.4105 E-07$ & $3.1999 E-06$ \\
& $L_{2}$ & $3.0418 E-08$ & $6.0835 E-08$ & $7.0812 E-07$ & $8.0355 E-06$ \\
\hline \multirow{2}{*}{ UAHT [37] } & $L_{\infty}$ & $6.0735 E-07$ & $5.8321 E-07$ & $6.2103 E-07$ & $6.2033 E-07$ \\
\hline \multirow{2}{*}{ UAH [37] } & $L_{2}$ & $4.4929 E-07$ & $5.7352 E-07$ & $2.1860 E-06$ & $2.1837 E-06$ \\
& $L_{\infty}$ & $8.0770 E-07$ & $1.3430 E-06$ & $2.5964 E-06$ & $1.5947 E-06$ \\
\hline \multirow{2}{*}{ UAT [37] } & $L_{2}$ & $6.2288 E-07$ & $1.0009 E-06$ & $2.1861 E-06$ & $1.5964 E-06$ \\
\hline
\end{tabular}

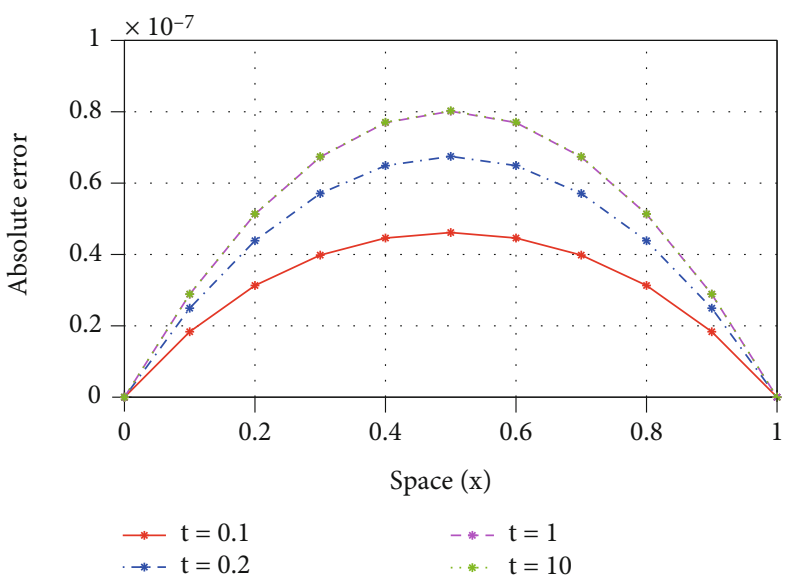

(a) Absolute error at different time levels

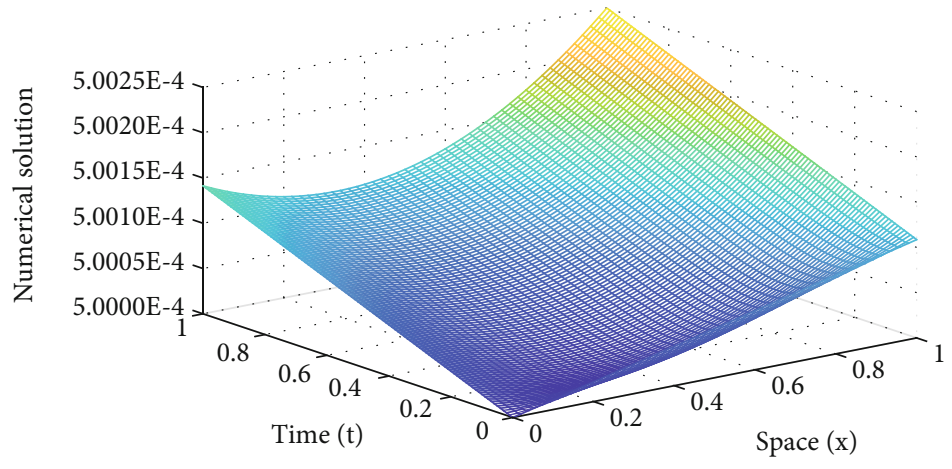

(b) Surface plot of numerical solution

FiguRE 6: Error and solution profile of Example 4 with $N=50$ and $\Delta t=0.01$.

where

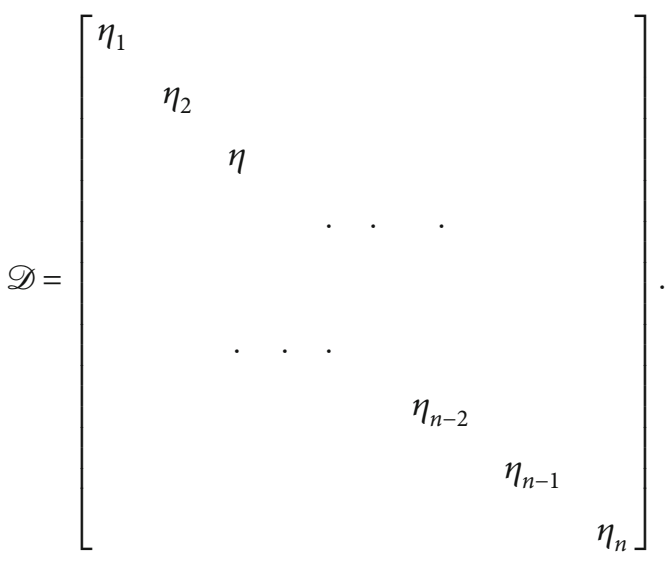

Taking $P^{-1} z=v$ in Equation (46), the differential equation becomes

$$
\frac{d v}{d t}=\mathscr{D} v
$$

Similarly, as discussed above, the solution of Equation (52) is $v=\exp (\mathscr{D} t) v^{0}$, and the recursive relation is

$$
v^{j+1}=E(\mathscr{D} \Delta t) v^{j}
$$

In this diagonal matrix, $E(\mathscr{D} \Delta t)$ is an approximate matrix of $\exp (\mathscr{D} \Delta t)$. The diagonal elements of the approximated matrix are $E^{j}\left(\eta^{j} \Delta t\right)$. Implementing Equation (25) on the scalar Equation (44),

$$
z^{\prime}=\eta^{j} z
$$

Thus, the method discussed in Equation (25) is absolutely stable if

$$
\left|E^{j}\left(\eta^{j} \Delta t\right)\right|<1
$$

where $\operatorname{Re}(\eta)<0$. The stability of the system exclusively depends on the eigenvalues of the coefficient matrix $\mathfrak{I}$ of the form $\sum_{\mathrm{m}=0}^{4}(\mathfrak{I} \Delta t)^{m} / m$ ! which should satisfy Equation (54). The necessary conditions that eigenvalues of $\mathfrak{I}$ should satisfy are given below [47]: 
TABLE 7: Comparison of absolute error of Example 5 with $\beta=0.1, \gamma=0.001, \eta=0.0001, \delta=2, h=0.1$, and $\Delta t=0.0001$.

\begin{tabular}{lcccccc}
\hline Method & & & & & $t=0.8$ \\
$x=0.5$ & $x=0.1$ & $x=0.9$ \\
\hline CFDM6 $(\Delta t=0.1)$ & $2.7448 E-12$ & $2.7405 E-12$ & $2.7442 E-12$ & $4.3917 E-12$ & $4.3848 E-12$ & $4.3908 E-12$ \\
EFD [49] & $4.3493 E-11$ & $1.2069 E-10$ & $4.3494 E-11$ & $4.3758 E-11$ & $1.2154 E-10$ & $4.3759 E-11$ \\
HSCM [50] & $2.1847 E-11$ & $6.0620 E-11$ & $2.1840 E-11$ & $2.1980 E-11$ & $6.1050 E-11$ & $2.1980 E-11$ \\
UAHT [37] & $7.3920 E-12$ & $2.0534 E-11$ & $7.3920 E-12$ & $7.3920 E-12$ & $2.0534 E-11$ & $7.3920 E-12$ \\
UAH [37] & $1.8881 E-11$ & $5.2390 E-11$ & $1.8881 E-11$ & $1.8998 E-11$ & $5.2769 E-11$ & $1.8998 E-11$ \\
UAT [37] & $1.8892 E-11$ & $5.2427 E-11$ & $1.8892 E-11$ & $1.8999 E-11$ & $5.2772 E-11$ & $1.8999 E-11$ \\
\hline
\end{tabular}

TABLE 8: Comparison of $L_{\infty}$ error norm of Example 5 with $\beta=0.1, \gamma=0.001, \eta=0.0001, h=0.1$, and $\Delta t=0.001$ for different values of $\delta$.

\begin{tabular}{|c|c|c|c|c|c|c|}
\hline \multirow{2}{*}{ Method } & \multicolumn{3}{|c|}{$t=0.2$} & \multicolumn{3}{|c|}{$t=1$} \\
\hline & $\delta=1$ & $\delta=4$ & $\delta=8$ & $\delta=1$ & $\delta=4$ & $\delta=8$ \\
\hline CFDM6 $(\Delta t=0.1)$ & $5.7337 E-13$ & $1.1345 E-12$ & $1.1825 E-12$ & $2.8669 E-12$ & $5.6727 E-12$ & $5.9123 E-12$ \\
\hline MCSCM [51] & $3.0271 E-13$ & $5.6344 E-10$ & $2.0904 E-09$ & $3.4889 E-13$ & $6.4937 E-10$ & $2.4085 E-09$ \\
\hline MGT [52] & $3.0804 E-13$ & $5.7325 E-10$ & $2.1267 E-09$ & $3.5806 E-13$ & $6.6634 E-10$ & $2.4720 E-09$ \\
\hline UAHT [37] & $1.3929 E-13$ & $2.5756 E-10$ & $9.5551 E-10$ & $1.4017 E-13$ & $2.5918 E-10$ & $9.6154 E-10$ \\
\hline UAH [37] & $3.0631 E-13$ & $5.7006 E-10$ & $2.1148 E-09$ & $3.5847 E-13$ & $6.6629 E-10$ & $2.4718 E-09$ \\
\hline UAT [37] & $3.0790 E-13$ & $5.7372 E-10$ & $2.1284 E-09$ & $3.5746 E-13$ & $6.6630 E-10$ & $2.4719 E-09$ \\
\hline
\end{tabular}

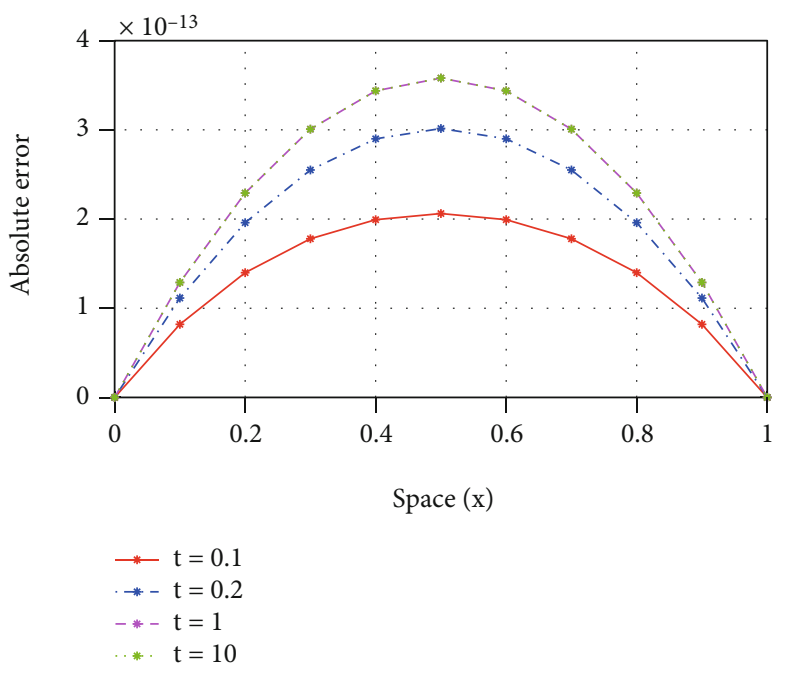

(a) Absolute error at different time levels

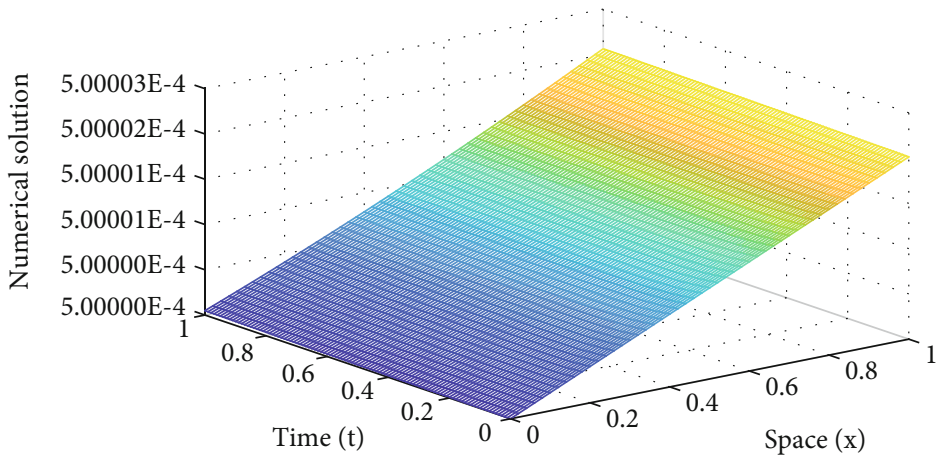

(b) Surface plot of numerical solution

FiguRE 7: Error and solution profile of Example 5 with $N=50$ and $\Delta t=0.01$.

(i) For real $\eta^{j}::-2.78<\Delta t \eta^{j}<0$

(ii) For pure imaginary $\eta^{j}:-2 \sqrt{2}<\Delta t \eta^{j}<2 \sqrt{2}$

(iii) For complex $\eta^{j}: \Delta t \eta^{j}$ should lie in the region as given by [48]

For different values of parameters, eigenvalues corresponding to gBF and $\mathrm{gBH}$ equations are given in Figures 1 and 2, respectively. It can be clearly observed that the eigenvalues of all the considered problems satisfy the above defined conditions; therefore, the proposed technique is unconditionally stable.

\section{Numerical Experiments}

The accuracy of compact finite difference scheme is measured using the $L_{2}$ and $L_{\infty}$ error norms, which are defined as follows:

$$
L_{\infty}=\max _{0 \leq i \leq N}\left|z_{i}-Z_{i}\right|, L_{2}=\sqrt{h \sum_{i=0}^{N}\left(z_{i}-Z_{i}\right)^{2}},
$$


TABLE 9: Comparison of absolute error of Example 6 with $\beta=5, \gamma=10, \eta=0.0001, \delta=2, h=0.1$, and $\Delta t=0.0001$.

\begin{tabular}{cccccc}
\hline$t$ & $x$ & CFDM6 $(\Delta t=0.1)$ & EFD [49] & HSCM [50] & UAHT [37] \\
\hline \multirow{3}{*}{0.2} & 0.1 & $1.2065 E-08$ & $6.58058 E-07$ & $1.971 E-07$ & $5.69755 E-08$ \\
& 0.5 & $1.2065 E-08$ & $1.78564 E-06$ & $1.971 E-07$ & $2.41380 E-07$ \\
\hline \multirow{3}{*}{0.5} & 0.9 & $1.2065 E-08$ & $6.58087 E-07$ & $2.233 E-07$ & $8.69729 E-08$ \\
\hline & 0.1 & $3.0158 E-08$ & $7.45354 E-07$ & $6.198 E-07$ & $2.74403 E-08$ \\
& 0.5 & $3.0158 E-08$ & $2.06834 E-06$ & $2.233 E-07$ & $8.74376 E-08$ \\
\hline 0.8 & 0.9 & $3.0159 E-08$ & $7.45392 E-07$ & $2.247 E-07$ & $8.74351 E-08$ \\
& 0.1 & $4.8246 E-08$ & $7.49483 E-07$ & $6.242 E-07$ & $2.42873 E-07$ \\
& 0.5 & $4.8247 E-08$ & $2.08190 E-06$ & $2.247 E-07$ & $8.74324 E-08$ \\
\hline
\end{tabular}

TABle 10: Comparison of absolute error of Example 6 with $\beta=5, \gamma=10, \eta=0.00001, \delta=2, h=0.1$, and $\Delta t=0.0001$.

\begin{tabular}{cccccc}
\hline$t$ & $x$ & CFDM6 $(\Delta t=0.1)$ & EFD [49] & HSCM [50] & UAHT [37] \\
\hline \multirow{3}{*}{0.2} & 0.1 & $1.2066 E-10$ & $2.08154 E-08$ & $6.235 E-09$ & $1.692 E-08$ \\
& 0.5 & $1.2066 E-10$ & $5.64806 E-08$ & $6.235 E-09$ & $7.63381 E-09$ \\
\hline \multirow{3}{*}{0.5} & 0.9 & $1.2066 E-10$ & $2.08155 E-08$ & $7.065 E-09$ & $2.75062 E-09$ \\
\hline & 0.1 & $3.0164 E-10$ & $2.35874 E-08$ & $1.960 E-08$ & $7.68188 E-09$ \\
& 0.5 & $3.0164 E-10$ & $6.54514 E-08$ & $7.065 E-09$ & $2.76547 E-09$ \\
\hline 0.8 & 0.9 & $3.0164 E-10$ & $2.35875 E-08$ & $7.108 E-09$ & $2.76547 E-09$ \\
& 0.1 & $4.8262 E-10$ & $2.37299 E-08$ & $1.974 E-08$ & $7.68186 E-09$ \\
& 0.5 & $4.8262 E-10$ & $6.59132 E-08$ & $2.108 E-09$ & $2.76546 E-09$ \\
\hline
\end{tabular}

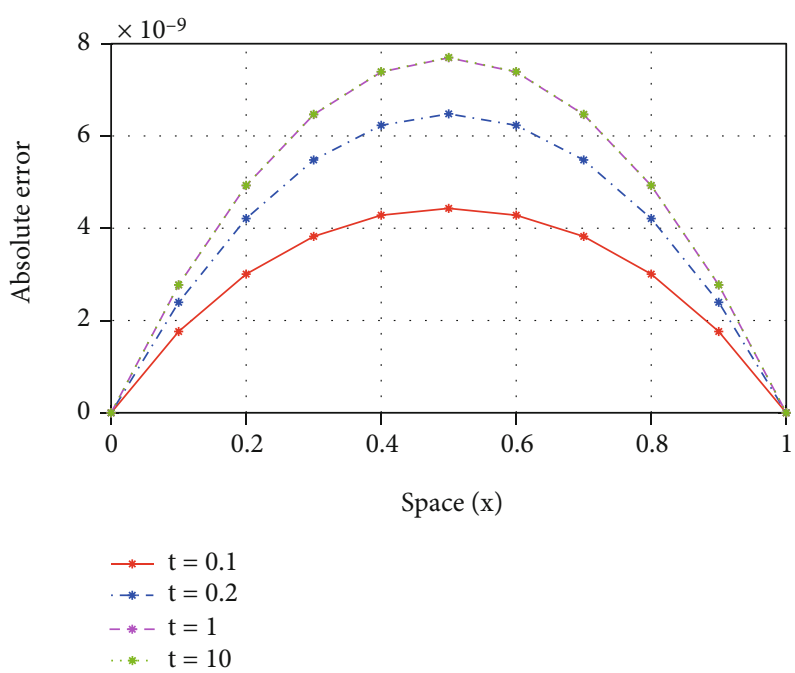

(a) Absolute error at different time levels

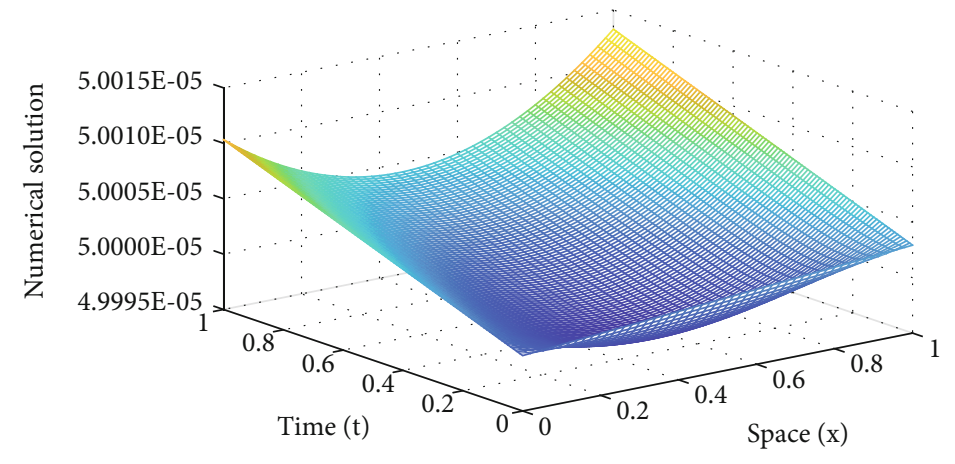

(b) Surface plot of numerical solution

FIGURE 8: Error and solution profile of Example 6 with $N=50$ and $\Delta t=0.01$.

where $z_{i}$ and $Z_{i}$ represent the exact and numerical solutions, respectively, at the node point $x_{i}$ for some fixed time.

Example 1. Consider gBF Equation (3) with the parameters $\beta=0.001$ and $\gamma=0.001$ for the initial condition as Equation (4) and the boundary conditions as (5) and (6). The exact solution is given by Equation (7). Table 1 gives a comparison of the absolute error for fixed spatial step size $h=0.1$ and temporal step size $\Delta t=0.0001$. Absolute error is calculated at time levels $t=0.001,0.010,100$ with $\delta=1$ and $\delta=4$. The results are found to be more accurate in comparison to the Adomian decomposition method [23], compact FDM [25], 
TABLE 11: Comparison of absolute error of Example 7 with $\beta=0, \gamma=1, \eta=0.001, \delta=2, h=0.1$, and $\Delta t=0.0001$.

\begin{tabular}{ccccccc}
\hline$t$ & $x$ & CFDM6 & ADM [23] & FDS4 [29] & GCG [30] & MCQDQM [31] \\
\hline \multirow{3}{*}{0.05} & 0.1 & $3.7491 E-08$ & $5.5890 E-07$ & $1.1176 E-06$ & $4.8110 E-07$ & $4.4924 E-07$ \\
& 0.5 & $3.7493 E-08$ & $5.5884 E-07$ & $1.1175 E-06$ & $3.9966 E-07$ & $1.0307 E-06$ \\
& 0.9 & $3.7494 E-08$ & $5.5877 E-07$ & $1.1174 E-06$ & $3.9240 E-07$ & $4.4917 E-07$ \\
\hline \multirow{3}{*}{0.10} & 0.1 & $7.4981 E-08$ & $1.1178 E-06$ & $2.2353 E-06$ & $1.0397 E-06$ & $6.6147 E-07$ \\
& 0.5 & $7.4984 E-08$ & $1.1177 E-06$ & $2.2350 E-06$ & $9.5823 E-07$ & $1.7107 E-06$ \\
& 0.9 & $7.4987 E-08$ & $1.1175 E-06$ & $2.2347 E-06$ & $9.5091 E-07$ & $6.6139 E-07$ \\
\hline \multirow{3}{*}{1.00} & 0.1 & $7.4953 E-07$ & $1.1175 E-05$ & $2.2353 E-05$ & $1.1021 E-05$ & $9.9267 E-07$ \\
& 0.5 & $7.4956 E-07$ & $1.0074 E-05$ & $2.2350 E-05$ & $1.1057 E-05$ & $2.7793 E-06$ \\
& 0.9 & $7.4959 E-07$ & $1.1173 E-05$ & $2.2347 E-05$ & $1.0841 E-05$ & $9.9260 E-07$ \\
\hline
\end{tabular}

TABLE 12: Comparison of absolute error of Example 7 with $\beta=0, \gamma=1, \eta=0.001, \delta=3, h=0.1$, and $\Delta t=0.0001$.

\begin{tabular}{lccccc}
\hline$t$ & $x$ & CFDM6 & ADM [23] & FDS4 [29] & MCSDQM [31] \\
\hline \multirow{3}{*}{0.05} & 0.1 & $3.7499 E-08$ & $1.9841 E-06$ & $3.9673 E-06$ & $1.5946 E-06$ \\
& 0.5 & $3.7499 E-08$ & $1.9837 E-06$ & $3.9665 E-06$ & $3.6584 E-06$ \\
& 0.9 & $3.7499 E-08$ & $1.9833 E-06$ & $3.9657 E-06$ & $1.5942 E-06$ \\
\hline \multirow{2}{*}{0.10} & 0.1 & $7.4996 E-08$ & $3.9681 E-06$ & $7.9346 E-06$ & $2.3479 E-06$ \\
& 0.5 & $7.4996 E-08$ & $3.9673 E-06$ & $7.9330 E-06$ & $6.0721 E-06$ \\
\hline \multirow{3}{*}{1.00} & 0.9 & $7.4996 E-08$ & $3.9665 E-06$ & $7.9314 E-06$ & $2.3475 E-06$ \\
\hline & 0.1 & $7.4962 E-07$ & $3.9663 E-05$ & $7.9346 E-05$ & $3.5221 E-06$ \\
& 0.5 & $7.4962 E-07$ & $3.9655 E-05$ & $7.9330 E-05$ & $3.8610 E-06$ \\
\end{tabular}

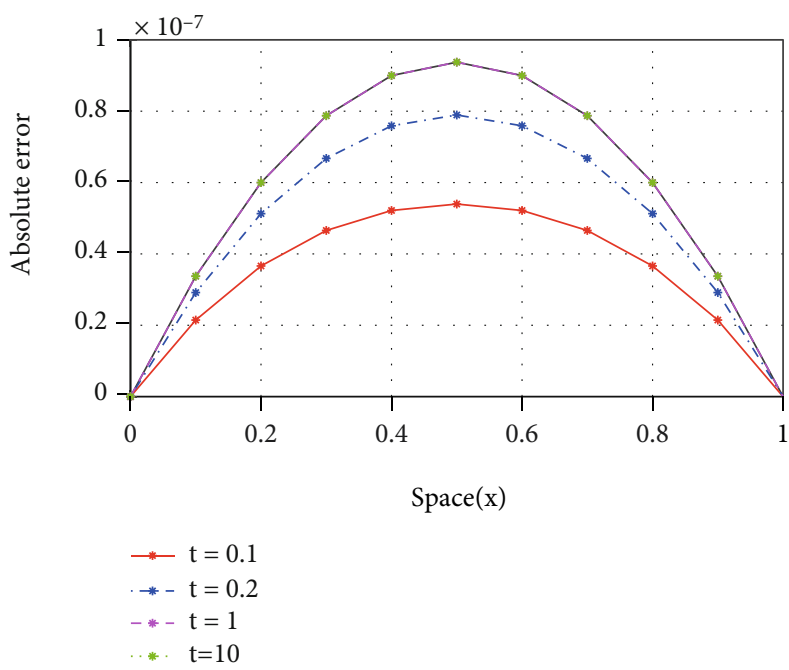

(a) Absolute error at different time levels

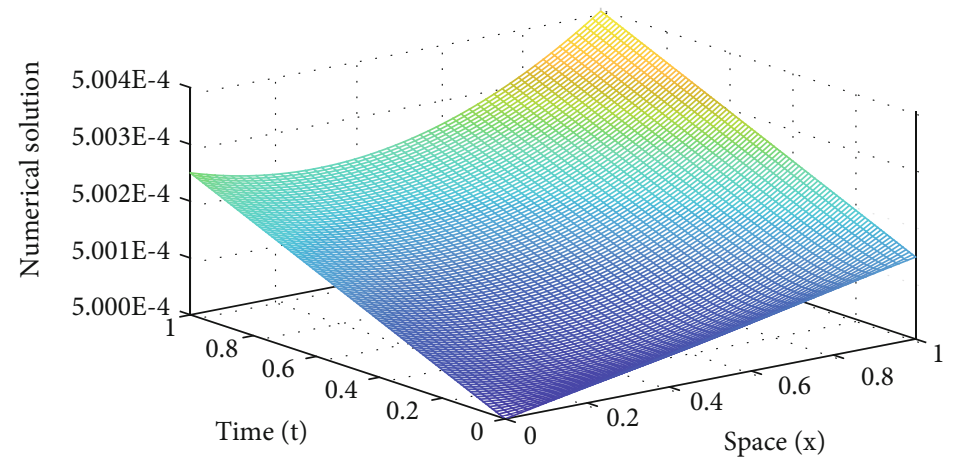

FIGURE 9: Error and solution profile of Example 7 with $N=50$ and $\Delta t=0.01$.

and exponential time differencing method of lines [29]. Figure 3(a) compares numerical and exact solution at different time levels, and Figure 3(b) presents the 3D behaviour of the numerical solution with $N=10, \Delta t=0.01$, and $\delta=8$.
Example 2. Consider Equation (3) for $\beta=\gamma=1$ with the initial condition (4) and boundary conditions (5) and (6). The absolute error is compared in Table 2 with those of previous investigators Ismail et al. [23], Sari et al. [25], and Bratsos 
[29] for $h=0.1, \Delta t=0.0001$, and $\delta=2,8$ at $t=0.0005$ and $t=0.0010$. Figure 4 (a) compares the numerical and exact solution at different time levels, and Figure 4(b) represents the $3 \mathrm{D}$ behaviour of numerical solution with $N=10, \Delta t=$ 0.01 , and $\delta=8$.

Example 3. Consider Equation (3) for the initial and boundary conditions (4) and (6) with $\beta=0.1$ and $\gamma=-$ 0.0025 . Table 3 depicts the accuracy of the results obtained by CFDM6, by comparing the absolute error with literature data for $h=0.1, \Delta t=0.0001$, and $\delta=2,4$, 8. Figure 5(a) compares the numerical and exact solution at different time levels, and Figure 5(b) represents the $3 \mathrm{D}$ behaviour of the numerical solution with $N=10, \Delta t$ $=0.01$, and $\delta=8$.

Example 4. Consider gBH Equation (8) with the initial and boundary conditions (9) and (10) for parametric values $\beta$ $=\gamma=1$ and $\eta=0.001$. The exact solution is given by (11). The absolute error at node points $x=0.1,0.5,0.9$ is given in Table 4 at $t=0.1$ and $t=1$ for $h=0.1, \Delta t=0.0001$, and $\delta=2$. Comparison shows that results are better than exponential finite difference scheme [49], hybrid B-spline [50], and tension B-spline collocation method [37]. Table 5 gives a comparison of $L_{\infty}$ error norm for $\delta=1,4$, and 8. Table 6 gives a comparison of $L_{2}$ and $L_{\infty}$ error norms with $\delta=2, h$ $=0.1, \eta=0.001, \Delta t=0.01$ at $t=0.05,0.1,1,5$. Figure 6(a) represents the absolute error at different time levels with $N$ $=10$, and Figure $6(\mathrm{~b})$ gives the $3 \mathrm{D}$ profile of numerical solution with $N=50, \Delta t=0.01$, and $\delta=8$.

Example 5. The gBH Equation (8) is considered for the initial and boundary conditions (9) and (10). The CFDM6 results are evaluated for $\beta=0.1$ and $\gamma=0.001$, and $\eta=0.0001$ $h=0.1, \Delta t=0.0001, \quad$ and $\delta=2$ at time $t=0.5$ and $t=0.8$ are given in Table 7 . The absolute error is compared with [37, $49,50]$. The $L_{\infty}$ error norm is compared for CFDM6 with the collocation of cubic B-splines [51], multiscale RungeKutta Galerkin method (MGT) [52], and a new kind of tension B-spline function [37] and is presented in Table 8 at different values of $\delta=1,4,8$. Figure 7(a) represents the absolute error at different time levels with $N=10$, and Figure 7 (b) gives the $3 \mathrm{D}$ profile of numerical solution with $N=50, \Delta t=0.01$, and $\delta=8$.

Example 6. Consider gBH Equation (8) with initial and boundary conditions (9) and (10). The absolute error is compared with the schemes discussed by $[37,49,50]$ for $\beta$ $=5, \gamma=10, \eta=0.0001, \Delta t=0.0001$, and $\delta=2$ at different node points for time $t=0.2,0.5$, and 0.8 . Tables 9 and 10 give a comparison of absolute error for $\eta=0.0001$ and $\eta=$ 0.00001 , respectively. Remarkable closeness of numerical and exact solutions can be seen in the tables. Figure 8(a) represents the absolute error at different time levels with $N=10$, and Figure $8(\mathrm{~b})$ gives the $3 \mathrm{D}$ profile of numerical solution with $N=50, \Delta t=0.01$, and $\delta=8$.
Example 7. The gBH Equation (8) is subjected to initial and boundary conditions (9) and (10) for $\beta=0, \gamma=1$, and $\eta=0.001$. Table 11 compares absolute error of CFDM6 with the Adomian decomposition method (ADM) [23], fourth-order numerical scheme (FDS4) [29], Gauss Chebyshev Galerkin (GCG) [30], and modified cubic B-spline differential quadrature method (MCSDQM) [31] at $\delta=2, h=0.1$, and $\Delta t=0.0001$. Table 12 gives the comparison of absolute error for $\delta=3$. The efficiency of the numerical solution to approach the exact solution can be easily seen, and the results are better than those of other methods. Figure 9(a) represents the absolute error at different time levels with $N=10$, and Figure 9(b) gives the $3 \mathrm{D}$ profile of the numerical solution with $N=50, \Delta t$ $=0.01$, and $\delta=8$.

\section{Conclusion}

Compact FDM along with the SSP-RK43 scheme has been implemented to solve gBF and gBH equations. Several examples of both the equations are successfully solved with the proposed technique. Absolute error and $L_{2}$ and $L_{\infty}$ error norms are calculated and compared with the previous results. The results with CFDM6 are found to be better than those with many techniques like the Adomian decomposition method, exponential time differencing method of lines, cubic B-spline collocation method, exponential finite difference scheme, hybrid B-spline collocation, tension B-spline collocation, multiscale Runge-Kutta Galerkin method, and modified cubic B-spline differential quadrature method. Comparison shows that the technique is providing highly accurate results with ease in implementation and less computational effort.

\section{Data Availability}

The complete data is in the manuscript.

\section{Conflicts of Interest}

The authors declare that they have no conflicts of interest.

\section{Acknowledgments}

Ms. Shallu is thankful to CSIR New Delhi for providing financial assistance in the form of JRF with File No. 09/797(0016)/2018-EMR-I.

\section{References}

[1] H. Bateman, "Some recent researches on the motion of fluids," Monthly Weather Review, vol. 43, no. 4, pp. 163-170, 1915.

[2] J. M. Burgers, "Mathematical examples illustrating relations occurring in the theory of turbulent fluid motion," Transactions of the Royal Netherlands Academy of Arts and Sciences, vol. 17, pp. 1-53, 1939.

[3] R. A. Fisher, "The wave of advance of advantageous genes," Annals of Eugenics, vol. 7, no. 4, pp. 355-369, 1937.

[4] S. Petrovskii and N. Shigesada, "Some exact solutions of a generalized Fisher equation related to the problem of biological 
invasion," Mathematical Biosciences, vol. 172, no. 2, pp. 73-94, 2001.

[5] H. Chen and H. Zhang, "New multiple soliton solutions to the general Burgers-Fisher equation and the KuramotoSivashinsky equation," Chaos, Solitons \& Fractals, vol. 19, no. 1, pp. 71-76, 2004.

[6] M. Sari, G. Gurarslan, and I. Dag, "A compact finite difference method for the solution of the generalized Burgers-Fisher equation," Numerical Methods for Partial Differential Equations, vol. 26, no. 1, pp. 125-134, 2010.

[7] T. Zhao, C. Li, Z. Zang, and Y. Wu, "Chebyshev-Legendre pseudo-spectral method for the generalised Burgers-Fisher equation," Applied Mathematical Modelling, vol. 36, no. 3, pp. 1046-1056, 2012.

[8] R. Mohammadi, "Spline solution of the generalized BurgersFisher equation," Applicable Analysis, vol. 91, no. 12, pp. 2189-2215, 2012.

[9] M. Tatari, B. Sepehrian, and M. Alibakhshi, "New implementation of radial basis functions for solving Burgers-Fisher equation," Numerical Methods for Partial Differential Equations, vol. 28, no. 1, pp. 248-262, 2012.

[10] S. A. Malik, I. M. Qureshi, M. Amir, A. N. Malik, and I. Haq, "Numerical solution to generalized Burgers-Fisher equation using exp-function method hybridized with heuristic computation," PLoS One, vol. 10, no. 3, article e0121728, 2015.

[11] O. P. Yadav and R. Jiwari, "Finite element analysis and approximation of Burgers-Fisher equation," Numerical Methods for Partial Differential Equations, vol. 33, no. 5, pp. 1652-1677, 2017.

[12] J. E. Macias-Diaz and A. E. Gonzalez, "A convergent and dynamically consistent finite-difference method to approximate the positive and bounded solutions of the classical Burgers-Fisher equation," Journal of Computational and Applied Mathematics, vol. 318, pp. 604-615, 2017.

[13] M. Soori, "The variational iteration method and the Homotopy perturbation method to the exact solution of the generalized Burgers-Fisher equation," Calculus of Variations and Partial Differential Equations, vol. 5, no. 8, pp. 19-26, 2018.

[14] A. G. Bratsos and A. Q. M. Khaliq, "An exponential time differencing method of lines for Burgers-Fisher and coupled Burgers equations," Journal of Computational and Applied Mathematics, vol. 356, pp. 182-197, 2019.

[15] B. Gurbuz and M. Sezer, "A modified Laguerre matrix approach for Burgers-Fisher type nonlinear equations," in Numerical Solutions of Realistic Nonlinear Phenomena, vol. 31 of Nonlinear Systems and Complexity, pp. 107-123, Springer International Publishing, Cham, Switzerland, 2020.

[16] J. Li, "Geometric properties and exact travelling wave solutions for the generalized Burger-Fisher equation and the SharmaTasso-Olver equation," Journal of Nonlinear Modeling and Analysis, vol. 1, no. 1, pp. 1-10, 2019.

[17] O. O. Onyejekwe, B. Minale, F. Habtamu et al., "Numerical discrete-domain integral formulations for generalized Burger-Fisher equation," Applied Mathematics, vol. 11, no. 3, pp. 137-145, 2020.

[18] H. Zhang, Y. Xia, and P. R. N'gbo, “Global existence and uniqueness of a periodic wave solution of the generalized Burgers-Fisher equation," Applied Mathematics Letters, vol. 121, pp. 1-7, 2021

[19] X. Y. Wang, Z. S. Zhu, and Y. K. Lu, "Solitary wave solutions of the generalised Burgers-Huxley equation," Journal of Physics
A: Mathematical and General, vol. 23, no. 3, pp. 271-274, 1990.

[20] A. L. Hodgkin and A. F. Huxley, "A quantitative description of membrane current and its application to conduction and excitation in nerve," The Journal of Physiology, vol. 117, no. 4, pp. 500-544, 1952.

[21] R. FitzHugh, Mathematical Models of Excitation and Propagation in Nerve, Biological Engineering, McGraw Hill, New York, NY, USA, 1969.

[22] O. Y. Yefimova and N. A. Kudryashov, "Exact solutions of the Burgers-Huxley equation," Journal of Applied Mathematics and Mechanics, vol. 68, no. 3, pp. 413-420, 2004.

[23] H. N. Ismail, K. Raslan, and A. Abd Rabboh, “Adomian decomposition method for Burger's-Huxley and Burger'sFisher equations," Applied Mathematics and Computation, vol. 159, no. 1, pp. 291-301, 2004.

[24] H. Gao and R. X. Zhao, "New exact solutions to the generalized Burgers-Huxley equation," Applied Mathematics and Computation, vol. 217, no. 4, pp. 1598-1603, 2010.

[25] M. Sari, G. Gurarslan, and A. Zeytinoglu, "High-order finite difference schemes for numerical solutions of the generalized Burgers-Huxley equation," Numerical Methods for Partial Differential Equations, vol. 27, no. 5, pp. 1313-1326, 2011.

[26] I. Celik, "Haar wavelet method for solving generalized Burgers-Huxley equation," Arab Journal of Mathematical Sciences, vol. 18, no. 1, pp. 25-37, 2012.

[27] R. Zhang, X. Yu, and G. Zhao, "The local discontinuous Galerkin method for Burger's-Huxley and Burger's-Fisher equations," Applied Mathematics and Computation, vol. 218, no. 17, pp. 8773-8778, 2012.

[28] R. Mohammadi, "B-spline collocation algorithm for numerical solution of the generalized Burgers-Huxley equation," Numerical Methods for Partial Differential Equations, vol. 29, no. 4, pp. 1173-1191, 2013.

[29] A. G. Bratsos, "A fourth order improved numerical scheme for the generalized Burgers-Huxley equation," American Journal of Computational Mathematics, vol. 1, no. 3, pp. 152-158, 2011.

[30] M. El-Kady, S. M. El-Sayed, and H. E. Fathy, "Development of Galerkin method for solving the generalized Burger's-Huxley equation," Mathematical Problems in Engineering, vol. 2013, no. $4,2013$.

[31] B. K. Singh, G. Arora, and M. K. Singh, "A numerical scheme for the generalized Burgers-Huxley equation," Journal of the Egyptian Mathematical Society, vol. 24, no. 4, pp. 629-637, 2016.

[32] S. Zibaei, M. Zeinadini, and M. Namjoo, "Numerical solutions of Burgers-Huxley equation by exact finite difference and NSFD schemes," Journal of Difference Equations and Applications, vol. 22, no. 8, pp. 1098-1113, 2016.

[33] M. Bukhari, M. Arshad, S. Batool, and S. M. Saqlain, "Numerical solution of generalized Burgers-Huxley equation using local radial basis functions," International Journal of Advanced and Applied Sciences, vol. 4, no. 5, pp. 1-11, 2017.

[34] J. E. Macias-Diaz, "A modified exponential method that preserves structural properties of the solutions of the Burgers-Huxley equation," International Journal of Computer Mathematics, vol. 95, no. 1, pp. 3-19, 2018.

[35] K. Gilani and U. Saeed, "CAS wavelet Picard technique for Burgers-Huxley and Burgers equation," International Journal of Applied and Computational Mathematics, vol. 4, no. 5, pp. 133-147, 2018. 
[36] S. C. Shiralashetti and S. Kumbinarasaiah, "Cardinal B-spline wavelet based numerical method for the solution of generalized Burgers-Huxley equation," International Journal of Applied and Computational Mathematics, vol. 4, no. 2, pp. 73-86, 2018.

[37] N. Alinia and M. Zarebnia, "A numerical algorithm based on a new kind of tension B-spline function for solving BurgersHuxley equation," Numerical Algorithms, vol. 82, no. 4, pp. 1121-1142, 2019.

[38] A. C. Loyinmi and T. K. Akinfe, "An algorithm for solving the Burgers-Huxley equation using the Elzaki transform," $S N$ Applied Sciences, vol. 2, no. 1, pp. 7-24, 2020.

[39] A. G. Kushner and R. I. Matviichuk, "Exact solutions of the Burgers-Huxley equation via dynamics," Journal of Geometry and Physics, vol. 151, article 103615, 2020.

[40] S. Shukla and M. Kumar, "Error analysis and numerical solution of Burgers-Huxley equation using 3-scale Haar wavelets," Engineering with Computers, vol. 1-9, 2020.

[41] S. Panghal and M. Kumar, "Approximate analytic solution of Burger Huxley equation using feed-forward artificial neural network," Neural Processing Letters, vol. 53, no. 3, pp. 21472163, 2021.

[42] S. K. Lele, "Compact finite difference schemes with spectrallike resolution," Journal of Computational Physics, vol. 103, no. 1, pp. 16-42, 1992.

[43] R. Ansari, R. Gholami, K. Hosseini, and S. Sahmani, “A sixthorder compact finite difference method for vibrational analysis of nanobeams embedded in an elastic medium based on nonlocal beam theory," Mathematical and Computer Modelling, vol. 54, no. 11-12, pp. 2577-2586, 2011.

[44] B. J. Boersma, “A 6th order staggered compact finite difference method for the incompressible Navier-Stokes and scalar transport equations," Journal of Computational Physics, vol. 230, no. 12, pp. 4940-4954, 2011.

[45] D. Li, C. Zhang, and J. Wen, "A note on compact finite difference method for reaction-diffusion equations with delay," Applied Mathematical Modelling, vol. 39, no. 5-6, pp. 17491754, 2015.

[46] R. Kaur, Shallu, V. K. Kukreja, N. Parumasur, and P. Singh, "Two different temporal domain integration schemes combined with compact finite difference method to solve modified Burgers' equation," Ain Shams Engineering Journal, 2021.

[47] M. K. Jain, Numerical Solutions of Differential Equations, Wiley Eastern Ltd., 1984.

[48] E. J. Kubatko, B. A. Yeager, and D. I. Ketcheson, "Optimal strong-stability-preserving Runge-Kutta time discretizations for discontinuous Galerkin methods," Journal of Scientific Computing, vol. 60, no. 2, pp. 313-344, 2014.

[49] B. Inan and A. R. Bahadir, "Numerical solutions of the generalized Burgers-Huxley equation by implicit exponential finite difference method," Journal of Applied Mathematics, Statistics and Informatics, vol. 11, no. 2, pp. 57-67, 2015.

[50] I. Wasim, M. Abbas, and M. Amin, "Hybrid B-spline collocation method for solving the generalized Burgers-Fisher and Burgers-Huxley equations," Mathematical Problems in Engineering, vol. 2018, Article ID 6143934, 18 pages, 2018.
[51] R. C. Mittal and A. Tripathi, "Numerical solutions of generalized Burgers-Fisher and generalized Burgers-Huxley equations using collocation of cubic B-splines," International Journal of Computer Mathematics, vol. 92, no. 5, pp. 1053-1077, 2015.

[52] J. Chen, "An efficient multiscale Runge-Kutta Galerkin method for generalized Burgers-Huxley equation," Applied Mathematical Sciences, vol. 11, no. 30, pp. 1467-1479, 2017. 